\title{
Spatial Fourier Transform for Blind Detection of Radio Observations of Rotating Radio Transients
}

Xiaoqian $\mathrm{He}$

Follow this and additional works at: https://researchrepository.wvu.edu/etd

\section{Recommended Citation}

He, Xiaoqian, "Spatial Fourier Transform for Blind Detection of Radio Observations of Rotating Radio Transients" (2018). Graduate Theses, Dissertations, and Problem Reports. 5787.

https://researchrepository.wvu.edu/etd/5787

This Thesis is protected by copyright and/or related rights. It has been brought to you by the The Research Repository @ WVU with permission from the rights-holder(s). You are free to use this Thesis in any way that is permitted by the copyright and related rights legislation that applies to your use. For other uses you must obtain permission from the rights-holder(s) directly, unless additional rights are indicated by a Creative Commons license in the record and/ or on the work itself. This Thesis has been accepted for inclusion in WVU Graduate Theses, Dissertations, and Problem Reports collection by an authorized administrator of The Research Repository @ WVU. For more information, please contact researchrepository@mail.wvu.edu. 


\title{
Spatial Fourier Transform for Blind Detection of Radio Observations of Rotating Radio Transients
}

\author{
Xiaoqian $\mathrm{He}$ \\ Thesis submitted to the \\ Benjamin M. Statler College of Engineering and Mineral Resources \\ at West Virginia University \\ in partial fulfillment of the requirements for the degree of \\ Master of Science \\ in \\ Electrical Engineering \\ Natalia Schmid, D.Sc., Chair \\ Kevin Bandura, Ph.D. \\ Maura McLaughlin, Ph.D.
}

Lane Department of Computer Science and Electrical Engineering

Morgantown, West Virginia

2018

Keywords: Radio Observation, Rotating Radio Transients (RRATs), Pulse Detection, Spatial Fourier Transform (SFT)

Copyright 2018 Xiaoqian $\mathrm{He}$ 


\begin{abstract}
Spatial Fourier Transform for Blind Detection of Radio Observations of Rotating Radio Transients
\end{abstract}

Xiaoqian $\mathrm{He}$

In this thesis we propose a new blind algorithm, which we call Energy in Transformed Data (ETD), for the detection of isolated astrophysical pulses. Unlike the conventional algorithm, which requires de-dispersion, integration over frequency channels and matched filtering, the ETD algorithm detects pulses in a space reciprocal to the space of filterbank data. The ETD algorithm is implemented in three steps: (1) it applies the Spatial Fourier Transform (SFT) to filterbank data, (2) it evaluates the energy of signal in the transformed space, and (3) it compares the value of the total energy to a threshold. If the energy in transformed data exceeds the threshold, we claim that we detect a potential pulse.

To analyze the detection capabilities of the ETD algorithm, we applied it to data of ten different Rotating Radio Transients (RRATs). The performance of the algorithm was compared to the performance of two other algorithms, one of which is the conventional algorithm and the other one is an algorithm that performs matched filtering in the SFT domain. The performance of the ETD algorithm depends on the Signal-to-Noise ratio (SNR) of pulses. When the SNR threshold is set to a large value such as 8 , the ETD algorithm can detect $80.5 \%$ of all pulses detected by the conventional algorithm. When the SNR threshold is set to 5, the ETD algorithm can detect only $40.4 \%$ detectable by the conventional algorithm.

The ability to detect broad and relatively weak astronomical pulses is a special feature of ETD. As we demonstrate it in this thesis, the energy of pulses with a broad pulse profile (broad compared to the time delay of the pulse arrival at the lower frequency of receiver) is concentrated around the origin in the SFT domain. This allows to easily detect broad pulses by ETD.

The main drawback of the ETD algorithm is in its inability to detect pulses which are relatively low in SNR value and have a narrow pulse profile. Analyzing the main advantages and disadvantages of ETD, we conclude that the algorithm can be used as a complementary method to the conventional algorithm, especially when the task is to detect broad pulses parameterized by a low SNR value. In addition to its use for current observations, we expect this method will be beneficial for detection of other types of isolated transients. 


\section{Acknowledgments}

Firstly, I would like to express my deepest gratitude to my advisor and committee Chair Dr. Natalia A. Schmid, who had morally supported, encouraged and provided invaluable advice during the past two years. Her passionate attitude to designing optimal systems and her knowledge of the subject inspired me in doing this research. Without her help this thesis would not be possible. I consider it a privilege to have her as my instructor of the Stochastic Systems class and to work on my research under her guidance.

I would also like to extend my gratitude to my committee members, Dr. Bandura and Dr. McLaughlin, who provided me with their feedback on how to improve my thesis. I would like to send my special thanks to Dr. Prestage of Green Bank Observatory for giving me a number of valuable suggestions regarding this project.

I would like to thank Brent Shapiro-Albert, my good collaborator. He provided benchmark data for the performance comparison presented in this thesis. His data were used in many tables and Figures 4.1, 4.2, 4.3, and 4.4.

I am grateful to all my lab mates, Zhicheng, Marwan, Ming and to all my friends, for their generous help and support.

Finally, I would like to thank my family, especially my parents and my husband who love, support and understand me. 


\section{Contents}

Acknowledgments

List of Figures vi vi v v v v

\begin{tabular}{ll}
\hline List of Tables & ix
\end{tabular}

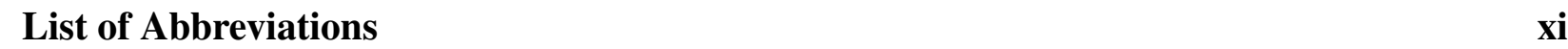

\begin{tabular}{lll|}
\hline & Introduction & 1
\end{tabular}

1.1 Main Goal . . . . . . . . . . . . . . . . . . . . . . . . . . . . 3

1.2 Data for performance analysis $\ldots \ldots \ldots \ldots \ldots$

1.3 Thesis Organization $\ldots \ldots \ldots \ldots \ldots \ldots$. . . . . . . . . . . . . . . 4

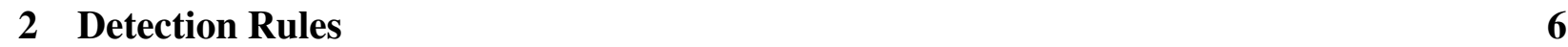

2.1 Basic Concepts . . . . . . . . . . . . . . . . . . . . 6

2.2 Algorithm 1: Energy in Transformed Data (ETD) $\ldots \ldots \ldots \ldots$

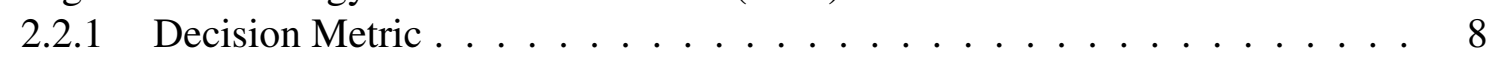

2.2 .2 Decision Rule . . . . . . . . . . . . . . . . . . . . . 11

2.3 Algorithm 2: Matched Filtering in SFT domain (MF-SFT) . . . . . . . . . . . . 11

2.3.1 Implementation of Matched Filters in the SFT Domain . . . . . . . . . . . 12

2.3 .2 Decision Metric . . . . . . . . . . . . . . . . . . 12

2.3 .3 Decision Rule . . . . . . . . . . . . . . . . . . . . . . 13

2.4 Algorithm 3: Conventional Algorithm (CA) . . . . . . . . . . . . . . . 14

3 Data 16

3.1 Data Used and Initial Data Processing . . . . . . . . . . . . . . . . . . . . 16

3.2 Timing Solution $\ldots \ldots \ldots \ldots \ldots \ldots \ldots \ldots$

\begin{tabular}{|lll}
\hline & Experimental Results & 22
\end{tabular}

4.1 Performance Comparison . . . . . . . . . . . . . . . . . . . . 22

$4.2 \quad$ SNR calculated by a Matched Filter with a Gaussian vs. Rectangular Profile . . . . 33

4.3 Candidate Pulses Detected in a Window Size of either $16 \times 16$ or $32 \times 32$, but not

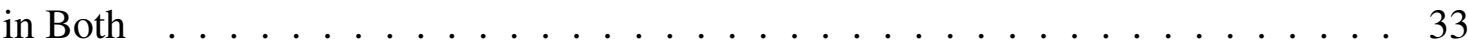

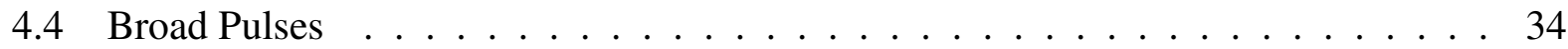


\begin{tabular}{|lll}
\hline 5 & Summary and Future Work & $\mathbf{4 7}$
\end{tabular}

5.1 Brief Summary $\ldots \ldots \ldots \ldots \ldots \ldots$. . . . . . . . . . . . . . . . . . . . . . . .

5.1.1 Conclusions regarding the relative performance of the ETD . . . . . . . . . 47

5.1 .2 Using the MF-SFT Algorithm to Confirm Candidates . . . . . . . . . . . . 48

5.2 Limitations of the ETD algorithm $\ldots \ldots \ldots \ldots \ldots$. . . . . . . . . . 48

5.3 Future Work . . . . . . . . . . . . . . . . . . . . . . . . . . . 49

\begin{tabular}{ll}
\hline Bibliography & $\mathbf{5 0}$
\end{tabular}

\begin{tabular}{|ll}
\hline A Appendix & 52
\end{tabular} 


\section{List of Figures}

1.1 Shown is a single pulse search output of a half hour observation (RR0037_0151.dat data file) of J1819-1548. The three upper panels (left to right) show the number of pulses versus SNR and DM channel, and the SNR versus DM channel. The panel at the bottom shows single bright pulses around DM channel of 300, which corresponds to the DM value of $196 \mathrm{pc} / \mathrm{cm}^{3}$. Note also the presence of the RFI peak at the DM value of $0 \mathrm{pc} / \mathrm{cm}^{3} .[1] \ldots \ldots \ldots \ldots{ }_{2}$

2.1 This is an example of a bright pulse of J1819-1458. Its spectrogram is shown in the top panel. The size of the spectrogram is $512 \times 2000(\mathrm{~K}=512, \mathrm{~N}=2000)$. The lower panel shows the result of applying a spatial Fourier transform (SFT) to the spectrogram. The characteristic signature of a pulse in the SFT domain is shown at the lower left corner and upper right corner. Zoomed in lower left corner is shown

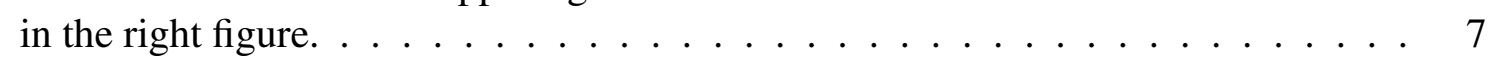

2.2 The left panels show three generated pulses parameterized by the DM values of 50, 150 , and $250 \mathrm{pc} / \mathrm{cm}^{3}$ without noise. The right panels show their SFT signatures. No bounding mask as in Equation $(2.5)$ is applied. . . . . . . . . . . . . 9

2.3 Two examples of a Spatial Fourier transformed spectrogram. The left panel shows the SFT signature of a pulse of J1819-1548. The right panel shows the SFT signature of noise $\ldots \ldots \ldots \ldots \ldots \ldots$

2.4 The left panels show spectrograms of three noise free dispersed pulses. The right panels show their three SFT signatures. The first row presents a pulse parameterized by $D M=196 \mathrm{pc} / \mathrm{cm}^{3}$ and $\mathrm{W}=5 \mathrm{~ms}$. The second row presents a pulse parameterized by $D M=196 \mathrm{pc} / \mathrm{cm}^{3}$ and $\mathrm{W}=25 \mathrm{~ms}$. The last row is a pulse generated with $D M=100 \mathrm{pc} / \mathrm{cm}^{3}$ and $\mathrm{W}=5 \mathrm{~ms} . \ldots \ldots \ldots 13$

2.5 The left panel shows the SFT signature of a noisy pulse of J1819-1458. The second panel shows a simulated SFT signature of a dispersed pulse with the same DM and width of J1819-1458, which is used as mask. The third figure shows the energy of the product of the two signatures. . . . . . . . . . . . . . . 14

2.6 Shown is a bright pulse of J1819-1458. The lower panel displays a spectrogram of a dispersed pulse. The upper panel displays its time series after dedispersion. . . 15

3.1 Choosing the size of the window for J1819-1458. Shown are seven windows. Each following window is shifted $0.04 \mathrm{sec}$ to the right or left of the previous window. . . 18 
3.2 Calculate TOA of potential pulses by adding $n \times$ Period to the TOA of a known pulse. For J1819-1458, a strong known pulse is located at 440.1873 sec. The period of this RRAT is $4.263 \mathrm{sec}$. By adding and subtracting one period at a time, we obtained the TOAs of potential pulses. Thus pulses detected at the locations $444.4503 \mathrm{sec}, 448.7133 \mathrm{sec}$ and so on. . . . . . . . . . . . . . . . . 18

4.1 Shown are several candidates detected by the conventional algorithm. The left panel shows an example of a composite pulse. The pulse can be seen as a vertical line in the dedispersed data on the left panel. The middle panel shows an example of noise. The right panel shows an example of RFI, which can be seen as an approximately diagonal line in dedispersed data. . . . . . . . . . . . . . 23

4.2 This figure shows different smoothing levels of a bright pulse. Smoothing level 0 means the data is not smoothed. We can see that this bright pulse is visible even when it is not smoothed. When the smoothing level is larger, the pulse is more visible and has a higher SNR. . . . . . . . . . . . . . . . . 24

4.3 This figure shows different smoothing levels of a weak pulse. The peak of pulse is not significant, when smoothing level is 0 to 2 . When the smoothing level is larger,

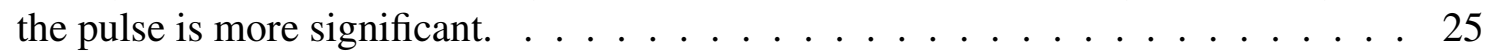

4.4 This figure shows different smoothing levels of white noise. There is no significant peak of pulse showing up from smoothing level 1 to 9. . . . . . . . . . . . . . 26

4.5 (a) Spectrogram of the pulse with the TOA at 645.3976 sec. in RR0090_0121 and SNR of 16.1. The pulse is detected by both the conventional algorithm and by the ETD algorithm. (b) Signature of the pulse in (a) in the SFT domain. (c) Spectrogram of the pulse at $661.5901 \mathrm{sec}$. in RR0090_0121 with SNR of 4.1. The pulse is detected by the ETD algorithm but not detected by the conventional algorithm. (d) The signature of this pulse in the SFT domain. . . . . . . . . . . . . 27

4.6 (a) Spectrogram of the pulse at 1408.0501 sec. in RR0086_0181 and SNR of 19.6. The pulse is detected by both the conventional algorithm and by the ETD algorithm. (b) Signature of this pulse in the SFT domain. (c) Spectrogram of the pulse at 1757.9401 sec. in RR0086_0181 with SNR of 2.8. The pulse is detected by the ETD algorithm but not detected by the conventional algorithm. (d) Signature of this pulse in the SFT domain. . . . . . . . . . . . . . . . 28

4.7 (a) Shown is a spectrogram of the pulse timed at 516.9901 sec. in RR0037_0071. The pulse is detected by both the conventional algorithm and by the ETD algorithm. (b) The signature of this pulse in the SFT domain. (c) Shown is a spectrogram of the pulse at 305.7601 sec. in RR0037_0071. The pulse is detected by the ETD algorithm but not detected by the conventional algorithm. (d) The signature of this pulse in the SFT domain. . . . . . . . . . . . . . . . . . . . 29

4.8 Two examples of a Fourier transformed spectrogram. Both figures show the SFT signature of a pulse of J1819-1548 arrived at 11005.3497 sec in RR0037_0151 data file. The left panel shows the SFT signature in only $16 \times 16$ window size, and the right panel shows the SFT signature in only $32 \times 32$ window size. $\quad \ldots . . . . .34$

4.9 The left plot shows a rectangular pulse $\mid$ A.1. The right plot shows its Fourier Trans-

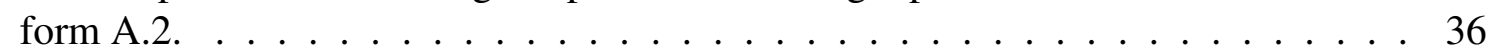


4.10 Panel (a) shows a spectrogram of the pulse timed at 440.1873 sec. in RR0037_0151. The pulse is detected by both the conventional algorithm and by the ETD algorithm. Panel (b) shows the signature of this pulse in the SFT domain. Panel (c) shows the spectrogram of the pulse at 423.0801 sec. in RR0037_0151. The pulse is detected by both the conventional algorithm and by the ETD algorithm. Panel (d) shows the signature of the pulse in (c) in the SFT domain. . . . . . . . . . . . . 37

4.11 Panel (a) shows the spectrogram of the pulse timed at 331.3901 s in RR0070_0131. The pulse is detected by both the conventional algorithm and by the ETD algorithm. Panel (b) displays the signature of this pulse in the SFT domain. Panel (c) shows the spectrogram of the pulse at $198.5001 \mathrm{~s}$ in RR0070_0131. The pulse is detected by only the ETD algorithm in $16 \times 16$ window size, but not detected by the conventional algorithm. Panel (d) displays the signature of this pulse in the SFT

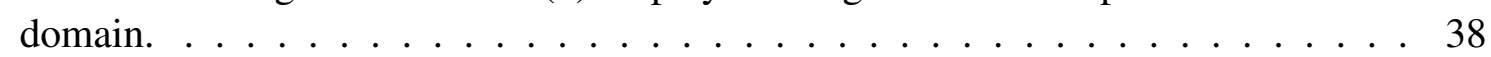

4.12 A plot of SNR values of pulses detected by the conventional algorithm versus the estimated width of each pulse. Blue diamonds indicate pulses detected by the ETD algorithm. Red squares indicate pulses missed by the ETD algorithm. Green triangles are pulses detected by the ETD algorithm but in a window of size $16 \times$ 16. Purple crosses mark pulses detected by the ETD algorithm in a window of size

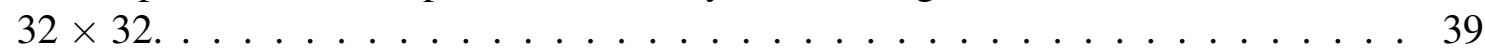




\section{List of Tables}

3.1 The observing parameters for ten RRATs include name, data file name, telescope, data machine, frequency, bandwidth, sample time, observation length, window length for each chunk, overlap between the chunk of candidates and the other chunks nearby, and the TOA of best pulse in each data file. The largest window length is $0.3 \mathrm{sec}$ for J1444-6026; the smallest window is of length $0.05 \mathrm{sec}$ for J1423-5647. List of time of best pulse is provided by our colleagues from the Department of Physics and Astronomy. . . . . . . . . . . . . . 20

3.2 Timing solutions of the conventional algorithm for the ten RRATs, including Right Ascension, Declination, spin period, period derivative, DM, epoch of period, and time delay. Width of pulse profile at $1400 \mathrm{MHz}$ is also listed in the sixth column. [2] 21

4.1 The second, third and forth columns present the number of candidate pulses detected by each individual algorithm. The fifth column lists the number of candidate pulses detected by both the conventional algorithm and by the ETD algorithm. The sixth column is the number of candidate pulses detected by both the ETD algorithm and by the algorithm performing Matched Filtering in the SFT domain. . . . . . . 30

4.2 The second column is the number of candidate pulses detected by the conventional algorithm after manual inspection. It means they are confirmed pulses. The third column presents the number of candidate pulses detected by the algorithms but not inspected by eye. The fourth column lists the number of candidate pulses which are not confirmed after being inspected by eye. They are considered as RFI and pulses due to noise. The fifth column presents the false alarm rate of the conventional algorithm for each data file. The last row of the table shows the total number of candidate pulses in the second through the fourth columns and the average false alarm rate. . . . . . . . . . . . . . . . . . . 31

4.3 The second column is the number of candidate pulses detected by the conventional algorithm with SNR exceeding 10, 9, 8, 7, 6, and 5. The third and fourth columns show how many candidate pulses, which have been detected by the conventional algorithm, are also detected by the ETD algorithm and by the algorithm implementing Matched Filtering in the SFT domain, respectively. The fifth and sixth columns present the percentage of detected candidate pulses by the ETD algorithm and by the algorithm implementing Matched Filtering in the SFT domain. . . . . . 32

4.4 List of candidate pulses of the conventional algorithm detected by ETD only in the SFT $16 \times 16$ matrix. SNR2 is SNR calculated for the rectangular pulse. . . . . . . . 35 
4.5 List of candidate pulses of the conventional algorithm detected by ETD only in the SFT $32 \times 32$ matrix. SNR2 is SNR calculated for rectangular pulse. . . . . . . . . . 35

4.6 The table presents a summary of the detection performance by the three detection algorithms described in the previous chapter. The table lists estimated width of pulse, number of candidate pulses, number of pulses detected by ETD, number of pulses not detected by ETD (neither in $16 \times 16$ nor $32 \times 32$ window size), number of pulses detected only in $16 \times 16$ window size, number of pulses detected only in $32 \times 32$ window size, detection rate of ETD, and detection rate if counting the pulses detected only in $16 \times 16$ or $32 \times 32$ window size. Each entry of the last row in the table is a sum of values along each column. The last two entries of the last row present the average of columns 7 and $8 . \ldots \ldots$. . . . . . . . . . 40

4.7 A summary of the detection performance by the three detection algorithms introduced in the previous chapter. The algorithms were applied to J1754-3011 data file of RR0037_0011. SNR2 is an SNR calculated using a rectangular pulse profile. 40

4.8 A summary of the detection performance by the three detection algorithms introduced in the previous chapter. The algorithms were applied to J1839-0136 data file of RR0037_0031. SNR2 is an SNR calculated using a rectangular pulse profile. 41

4.9 A summary of the detection performance by the three detection algorithms introduced in the previous chapter. The algorithms were applied to J1317-5759 data file of RR0037_0071. SNR2 is an SNR calculated using a rectangular pulse profile. 42

4.10 A summary of the detection performance by the three detection algorithms introduced in the previous chapter. The algorithms were applied to J1819-1458 data file of RR0037_0151. SNR2 is an SNR calculated using a rectangular pulse profile. 43

4.11 A summary of the detection performance by the three detection algorithms introduced in the previous chapter. The algorithms were applied to J1423-5647 data file of RR0070_0131. SNR2 is an SNR calculated using a rectangular pulse profile. 44

4.12 The performance of the three algorithms for J1913+1330 data file RR0086_0181's candidate pulses. SNR2 is SNR calculated for rectangle pulse profile. . . . . . . . 44

4.13 The performance of the three algorithms for J1826-1419 data file RR0090_0121's candidate pulses. SNR2 is SNR calculated for rectangle pulse profile. . . . . . . . 45

4.14 The performance of the three algorithms for J1444-6026 data file RR0090_0181's candidate pulses. SNR2 is SNR calculated for rectangle pulse profile. . . . . . . . 46

4.15 The performance of the three algorithms for J1846-0257 data file RR0090_0211's candidate pulses. SNR2 is SNR calculated for rectangle pulse profile. . . . . . . . 46

4.16 The performance of the three algorithms for J1739-2513 data file RR0090_0271's candidate pulses. SNR2 is SNR calculated for rectangle pulse profile. . . . . . . 46 


\section{List of Abbreviations}

RRATs Rotating radio transients

ISM interstellar medium

SFT Spatial Fourier transform

ECD energy in correlated data

FFT Fast Fourier Transform

SNR Single-to-Noise Ratio

GPUs graphics processing units

ETD Energy in Transformed Data

CA Conventional Algorithm

MF-SFT Matched Filtering in Spatial Fourier Transform domain

DM Dispersion Measure

TOA time-of-arrival

TOAs time-of-arrivals

RFI Radio Frequency Interference

RA right ascension

Dec. declination

GBT Green Bank Telescope 
PSNR peak signal-to-noise ratio

FAR false alarm rate

RRAT rotating radio transient 


\section{Chapter 1}

\section{Introduction}

Rotating radio transients ( $\mathrm{RRATs}$ ) are a special category of pulsars. Unlike pulsars, rotating magnetized neutron stars [3], RRATs emit energy sporadically. RRATs were discovered through a single pulse search of the Parkes Multibeam Pulsar Survey data (see McLaughlin et al. 2006 [4]). Since then they are objects of intense study, see for example publications by Keith et al. 2009 [5] and Keane et al. 2011 [6]. Figure 1.1] shows a plot of Dispersion Measure (DM) versus time which is traditionally used to inspect single pulses. A bright single pulse can be noticed around DM channel of 300 . We can also notice a peak at the DM value of $0 \mathrm{pc} / \mathrm{cm}^{3}$, which is an indicator of strong Radio Frequency Interference $(\underline{\mathrm{RFI}})$, a signal originated on the Earth. RRATs are known as a subgroup of rotating magnetized neutron stars. Typical values of the rotational period of RRATs are from 0.1 to $7 \mathrm{sec}$ [2]. As stated earlier RRATs emit energy sporadically so that their burst rates vary from one per minute to less than one per hour (see for details Keane \& McLaughlin 2011 [6]). Standard periodicity searches applied in radio astronomy for detection of new pulsars are based on Fast Fourier Transform (FFT) which is applied to time series data. The transformed data are then folded at a certain period to enhance Single-to-Noise Ratio $(\underline{\mathrm{SNR}})$ and profile of the pulse (Larsson 1996 [7]]).

Since their discovery RRATs have been the subject of continuous analysis. The first X-ray counterpart to a rotating radio transient ( $(\overline{R R A T}), \mathrm{J} 1819-1458$, was discovered by Chandra observation. The X-ray properties of CXOU J181934.1-145804 suggested that it was the same object as RRAT J1819-1458 and it was very likely to be a cooling neutron star. RRATs were thought to be a new class of neutron stars (Reynolds $2006[8]$ ). To figure out the connection and difference 
File: RR0037_0151.dat 107891 events. $\max \mathrm{S} / \mathrm{N}=69.0$. Peak $\mathrm{DM}=3.0 \mathrm{~cm} \backslash \mathrm{u}-3 \backslash \mathrm{d} p \mathrm{pc}$
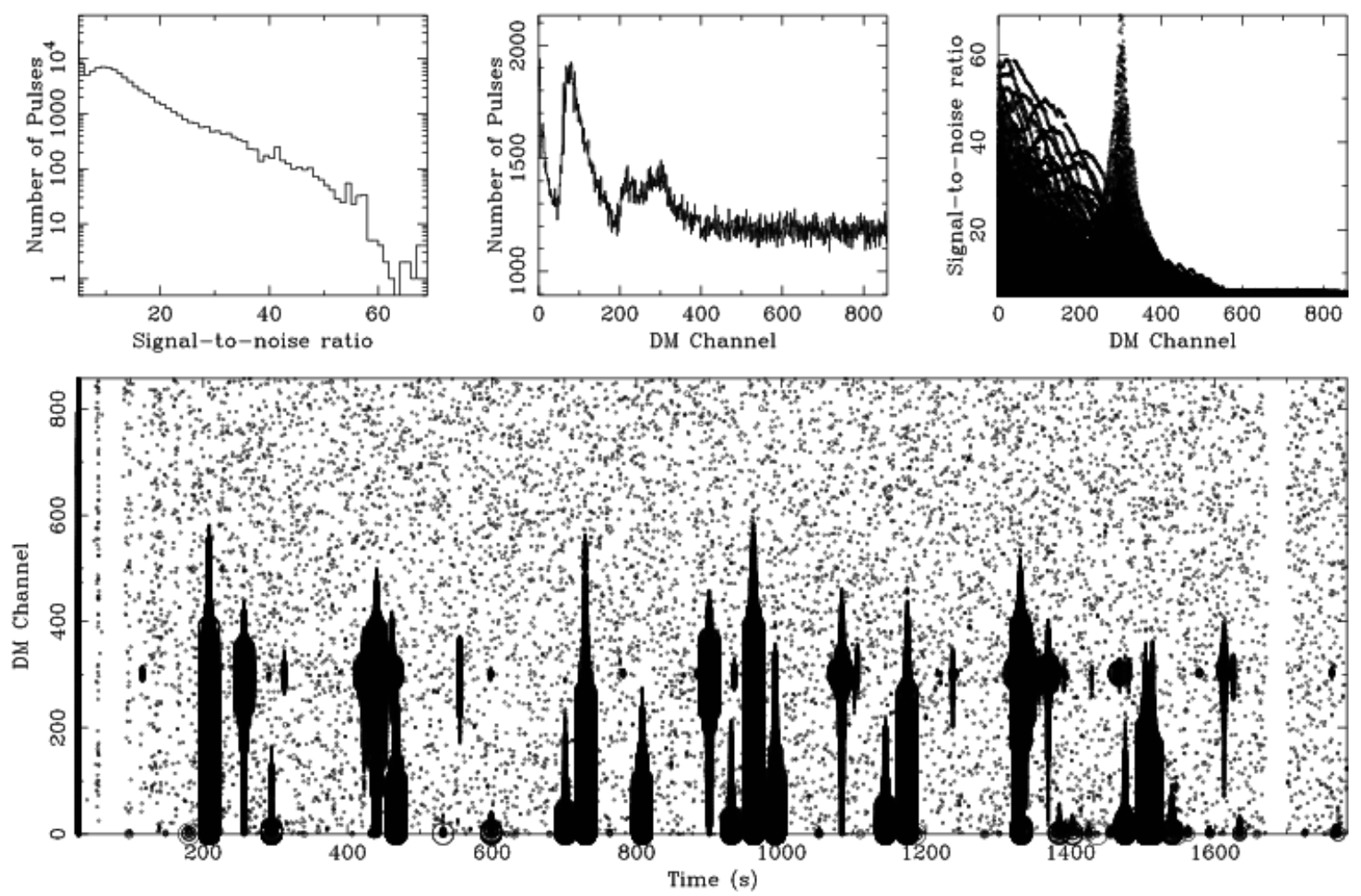

Figure 1.1: Shown is a single pulse search output of a half hour observation (RR0037_0151.dat data file) of J1819-1548. The three upper panels (left to right) show the number of pulses versus SNR and DM channel, and the SNR versus DM channel. The panel at the bottom shows single bright pulses around DM channel of 300, which corresponds to the DM value of $196 \mathrm{pc} / \mathrm{cm}^{3}$. Note also the presence of the RFI peak at the DM value of $0 \mathrm{pc} / \mathrm{cm}^{3}$. [1] 
between RRATs and other neutron stars, RRATs characteristics and X-ray properties of CXOU J181934.1-145804 were compared with the multiwavelength properties of other classes of neutron stars. After observing the differences of P-P distribution and birthrate (Burgay 2007 [9]) it was concluded that RRATs are not magnetars.

During the last decade various interpretations were presented to explain the sporadic nature of the energy emission by RRATs, There was a suggestion saying that a surrounding debris disk interacted with the magnetosphere of neutron star and quenched the radio emission. Transient radio emissions were allowed only temporarily when the disk did not go through the light cylinder (Li 2006 [10]). In addition, radiation belts might trap plasma in a certain density. When the trapped plasma got excited and waves disrupted the radiation belts, the plasma was released and radio bursts were produced (Luo \& Melrose 2007 [11]). Also asteroidal material injected into magnetosphere would affect the density of plasma and altitude of emission, which would let some emissions be intermittently detectable (Cordes \& Shannon 2008 [12]).

On the other hand, RRATs might be too far away from observation or too weak so that some emissions could not be detected and made RRATs emission behavior different from normal pulsars. It was suggested that longer observing time would be able to reveal this conjecture (Weltevrede et al. $2006[13])$.

\subsection{Main Goal}

The main goal of this thesis is to develop a novel transform-based algorithm for the detection of isolated astrophysical pulses and to demonstrate its performance on time series of several RRATs The performance of the algorithm will be compared to the performance of the conventional algorithm developed by McLaughlin et al. [4]. The conventional algorithm is the one which is currently applied in practice.

Our algorithm will use filterbank data (spectrograms) as its input. Filterbank data can be interpreted as images (the displayed function is an energy of the pulse across a number of discrete channels vs time; due to the properties of the interstellar medium (ISM) the signal first arrives to high frequencies of a receiver bandwidth, it will arrive to lower frequencies with a delay).

The conventional detection of pulses involves a number of basic steps: (1) data are de-dispersed, 
that is, the energy of the signal in different channels is vertically aligned, (2) the energy of the signal is integrated across frequency channels resulting in a one dimensional signal of the pulse as a function of time, (3) a filter with impulse response matched to the signal is applied to the one dimensional signal and (4) the peaks of the signal above the threshold of 5 of the noise are marked as potential pulses.

The new algorithm developed and presented in the next chapter is an alternative to the conventional detection algorithm for the detection of relatively bright pulses (high in SNR values). In the case of a low value of SNR it can be viewed as a complimentary approach with an ability to easily detect broad pulses while the conventional approach is sensitive to the detection of narrow pulses.

\subsection{Data for performance analysis}

In this thesis, we applied our algorithm to ten RRATs, They are J1317-5759, J1423-5647, J1444-6026, J1739-2513, J1754-3011, J1819-1458, J1826-1419, J1839-0136, J1846-0257, and J1913+1330. The listed RRATs were discovered while analyzing Parkes Multibeam Pulsar Survey data (Manchester et al. 2001 [14]; McLaughlin et al. 2006 [4]; Keith et al. 2009 [5]). The Parkes Observatory is an radio observatory in Australia with a dish of 64 meter in diameter. Since 1961 when it was built, the Parkes Observatory provided data supporting discoveries of quasars, interstellar magnetic fields and most of the known pulsars [15].

\subsection{Thesis Organization}

The rest of this thesis is organized as follows:

- Chapter 2 describes three blind algorithms used to detect isolated transient pulses. Among these algorithms, two are newly designed and the third one is the conventional algorithm currently used by radio astronomers.

- Chapter 3 describes data used to analyze performance of the algorithms. It also presents the experimental design and data pre-processing steps. 
- Chapter 4 compares the performance of the Conventional Algorithm ( $(\overline{\mathrm{CA}})$, Energy in Transformed Data (ETD) and the algorithm implementing Matched Filtering in Spatial Fourier Transform domain (MF-SFT).

- Chapter 5 presents conclusions regarding the performance of the algorithms and discusses the future work. 


\section{Chapter 2}

\section{Detection Rules}

In this chapter we describe three algorithms for detection of dispersed astrophysical pulses. One of them is the conventional approach and the other two are newly developed algorithms. We will begin with a description of new algorithms, but prior to this we will introduce a few relevant quantities.

\subsection{Basic Concepts}

Dispersion: An astrophysical pulse is broadband in its nature. It disperses as it travels through the ISM, which is filled with free electrons. As a result, low frequencies describing the pulse will be delayed compared to its high frequencies. This is a phenomenon known as dispersion. The time delay, $\Delta t$, experienced by an astrophysical pulse between a high frequency and a low frequency is described by the following equation:

$$
\triangle t=4.149 \times 10^{3} \times D M \times\left(\frac{1}{f_{\text {low }}^{2}}-\frac{1}{f_{\text {high }}^{2}}\right) \quad[\text { in sec. }],
$$

where $\overline{\mathrm{DM}}$ is the Dispersion Measure, the integrated column of free electrons over the line sight measured in $\mathrm{pc} / \mathrm{cm}^{3}$ and $f_{\text {low }}$ and $f_{\text {high }}$ are the low and high frequency in MHz. For example, the L-band receiver installed on the Green Bank Telescope (GBT) has a receiver with $f_{\text {low }}$ set to 720 $\mathrm{MHz}$ and $f_{\text {high }}$ set to $920 \mathrm{MHz}$. Denoting the time of arrival to the lowest frequency of a receiver 

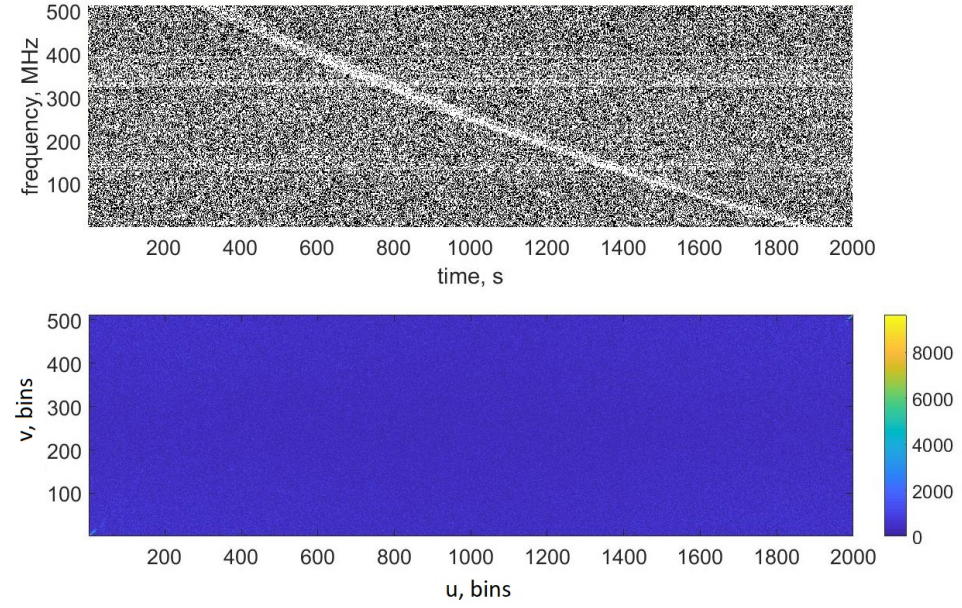

(a) Spectrogram and SFT

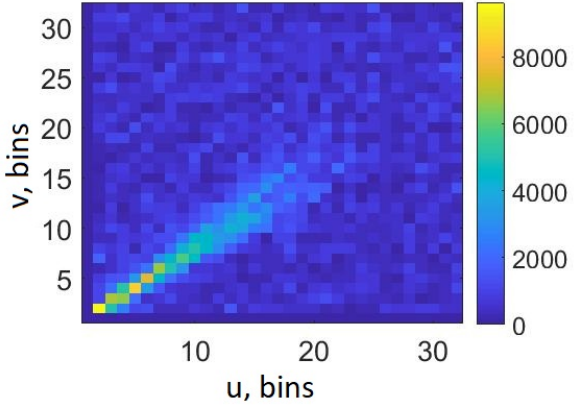

(b) Lower left corner of SFT

Figure 2.1: This is an example of a bright pulse of J1819-1458. Its spectrogram is shown in the top panel. The size of the spectrogram is $512 \times 2000(\mathrm{~K}=512, \mathrm{~N}=2000)$. The lower panel shows the result of applying a spatial Fourier transform (SFT) to the spectrogram. The characteristic signature of a pulse in the SFT domain is shown at the lower left corner and upper right corner. Zoomed in lower left corner is shown in the right figure.

as reference time, $t_{r e f}$, we can rewrite 2.1 for the delay in any frequency channel $f$ :

$$
t_{r e f}-t=4.149 \times 10^{3} \times D M \times\left(\frac{1}{f_{r e f}^{2}}-\frac{1}{f^{2}}\right) \quad[\text { in sec } .] .
$$

Spectrogram: Astronomical pulses observed by a telescope are time series sampled at extremely high frequency. The data are first cleaned of RFI and then transformed by means of a short time Fourier transform. The adjacent transformed time intervals are arranged along columns of a two dimensional array. To be more specific, a first interval composed of $K$ temporal samples of a received voltage signal, when Fourier transformed, is displayed as the first column of a two dimensional array. Next $K$ temporal samples are transformed and placed in the two-dimensional array as a second column, and the process continues until the end of time series is reached. The entries along each column are discrete frequency channels. The two-dimensional array is then passed through a squarer, which computes pixel-wise power of the two dimensional signal [16]. This array with $K$ channels and $N$ temporal bins is known as a spectrogram or filterbank data. An example of a typical spectrogram is displayed at the top of Figure 2.1.

Pulse Parameters: Each dispersed astrophysical pulse displayed in the form of a spectrogram is 
a two-dimensional function of time and frequency. In addition to this, each pulse can be described by a number of parameters including the pulse profile in each frequency channel. A typical pulse profile may be modeled as a Gaussian pulse or as a Gaussian function convolved with an exponential function. The width of the pulse varies widely. It can be as small as $0.1 \mathrm{~ms}$ in width and as broad as $100 \mathrm{~ms}$ in width. Both the width and the shape of the pulse profile affect the signature of the pulse in the Spatial Fourier transform (SFT) domain.

Spatial Fourier Transform (SFT): Let $g(t, f)$ denote a spectrogram. The SFT of the spectrogram, denote it $G(u, v)$, is given as

$$
G(u, v)=\sum_{t=0}^{N-1} \sum_{f=0}^{K-1} g(t, f) \exp \left(-j \frac{2 \pi t u}{N}\right) \exp \left(-j \frac{2 \pi f v}{K}\right),
$$

where $u$ and $v$ are known in image processing as spatial frequencies [16] and in astronomy as reciprocals of $t$ and $f$. The dimensions of the transformed spectrogram, $G(u, v)$ are the same as the dimensions of $g(t, f)$, that is, $K \times N$. As an illustration, a strong dispersed pulse and its SFT are shown in Figure 2.1. Note that due to parameters of the pulse (it is a relatively broad pulse compared to the time delay $\Delta t$ between the highest and lowest frequencies) the energy of SFT pulse is concentrated around the $(0,0)$ point in the SFT domain. The SFT signature of this pulse is shown in Figure 2.3 .

From (2.1), for a given number of frequency channels and a given number of temporal bins (samples) in a spectrogram, increasing the value of DM results in increasing the delay between the lowest and the highest frequencies. In the SFT domain increasing the $\overline{\mathrm{DM}}$ value of the pulse rotates its $\mathrm{SFT}$ signature counterclockwise. As an example, three dispersed pulses generated at DM $=50$, $150,250 \mathrm{pc} / \mathrm{cm}^{3}$ are displayed in Figure 2.2. The width of dispersed pulses was set to $5 \mathrm{~ms}$ and the shape profile is selected to be a rectangle.

\subsection{Algorithm 1: Energy in Transformed Data (ETD)}

\subsubsection{Decision Metric}

Let $R(u, v)$ denote the SFT of a noisy spectrogram. To detect a pulse in $R(u, v)$, we zoom on a small area of the $(u, v)$ space around $(0,0)$. The size of the area is determined by the width of 

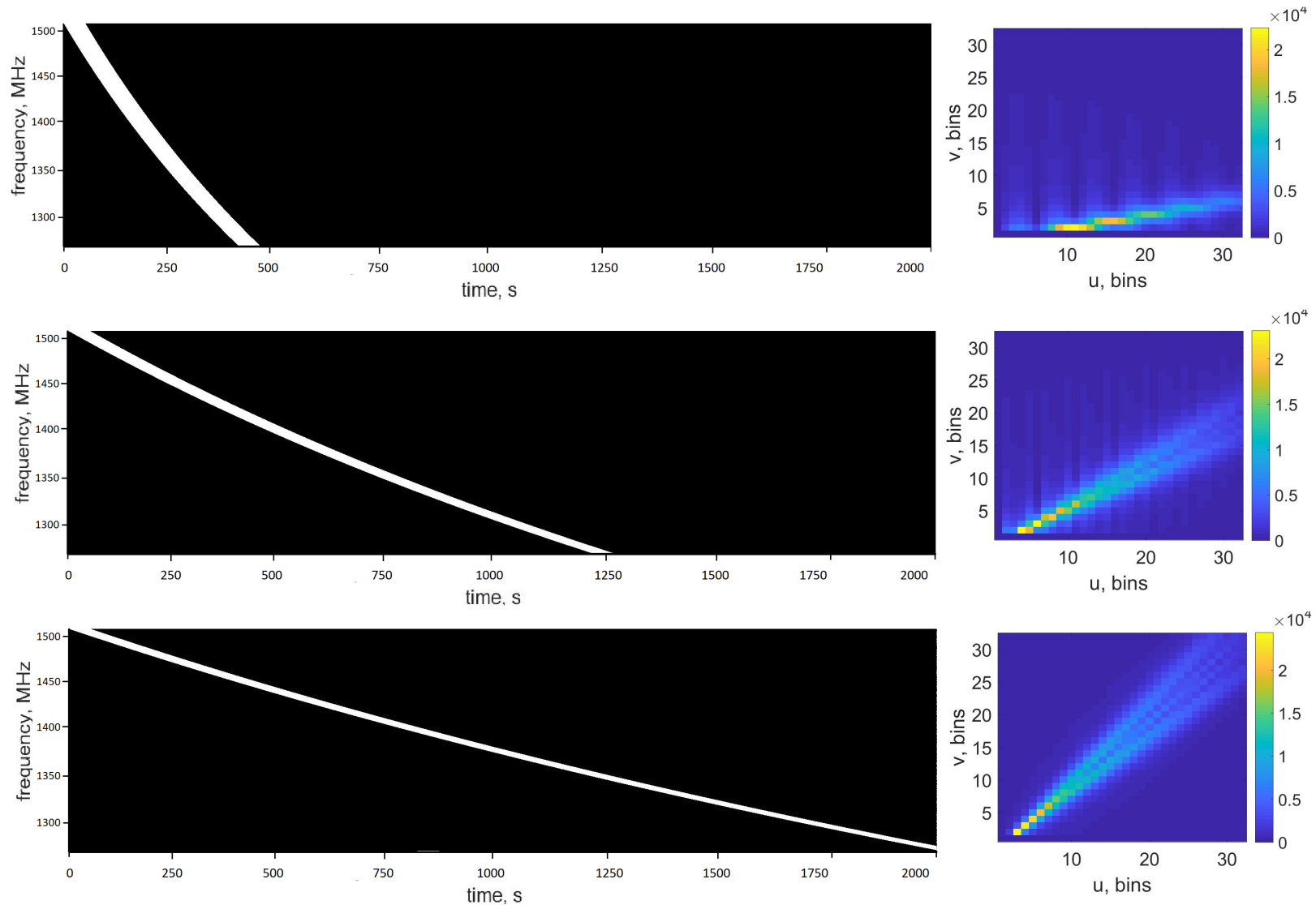

Figure 2.2: The left panels show three generated pulses parameterized by the DM values of 50, 150 , and $250 \mathrm{pc} / \mathrm{cm}^{3}$ without noise. The right panels show their SFT signatures. No bounding mask as in Equation 2.5 is applied.

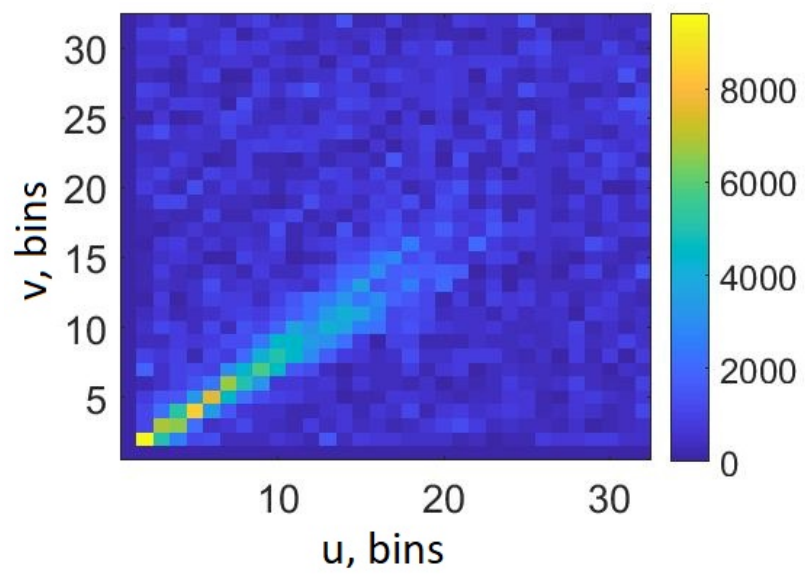

(a) SFT of a noisy pulse

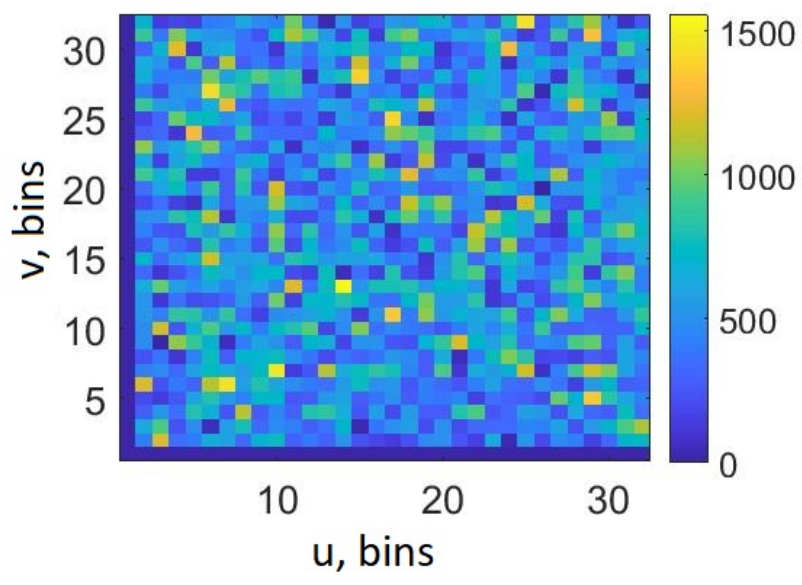

(b) SFT of noise

Figure 2.3: Two examples of a Spatial Fourier transformed spectrogram. The left panel shows the SFT signature of a pulse of J1819-1548. The right panel shows the SFT signature of noise. 
the pulse, denote it as $W$. In our numerical evaluation, for typical time and frequency resolutions, the area is set to maxval $\times$ maxval pixels with maxval varied from 16 to 32 . The choice of the two values results from the assumption that the width $W$ of the pulse is between $1 \mathrm{~ms}$ and $15 \mathrm{~ms}$, and the DM values are in the range between 30 and $300 \mathrm{pc} / \mathrm{cm}^{3}$. We defer the mathematical explanation of the choice of maxval to Appendix.

The DC component $(u, v)=(0,0)$ as well as the vertical and horizontal axes $u=0$ and $v=0$ are set to zero for $R(u, v)$. This ensures the removal of persistent RFI that show up in a spectrogram in the form of horizontal or vertical lines or bands.

As a performance metric we choose the Energy in Transformed Data (ETD) defined as:

$$
d_{E T D}=\sum_{\{(u, v): 0<u, v<\text { maxval }\}}|R(u, v:)|^{2} .
$$

To reduce the effect of noise in some (but not in all) cases the sum in 2.4 is evaluated only over the discrete values of $(u, v)$ satisfying the following double inequality:

$$
\frac{2 d}{f_{\text {high }}^{3}} \leq \frac{v}{u} \leq \frac{2 d}{f_{\text {low }}^{3}},
$$

where $d=4.149 \times 10^{3} \times D M$. The double inequality 2.5 is a bounding "fan" defined by the tangent lines to a dispersed pulse at $f_{\text {low }}$ and $f_{\text {high }}$. As the DM value increases, the "fan" rotates counter-clock wise around the origin in the SFT space.

In addition to the bounding box, we include an option that allows selecting "informative" entries in $|R(u, v)|^{2}$. The selection is justified by the Stein's principle [17] of estimation of the mean of a multivariate normal distribution, which underlies the theory of wavelet shrinkage by Donoho and Johnstone [18]. They argue that wavelet coefficients above a universal asymptotic threshold contribute information to the representation of a signal in terms of wavelets, while the coefficients below the threshold are attributed to noise. The asymptotically optimal threshold by Donoho and Johnstone is given as $\sigma^{2} \sqrt{2 \log (K N)}$, where $\sigma^{2}$ is the noise variance in the SFT domain.

Denote by $\Phi_{\{x\}}$ an indicator function of an event $x$, that is $\Phi_{\{x\}}=1$, if the event $x$ holds and $\Phi_{\{x\}}=0$, otherwise. Thus, the metric $(2.4)$ constrained by the two conditions above can be reduced 
to

$$
d_{E T D}=\sum_{\left\{(u, v): \frac{2 d}{f_{\text {high }}^{3}} \leq \frac{v}{u} \leq \frac{2 d}{f_{\text {low }}^{3}}\right\}}|R(u, v)|^{2} \times \Phi_{\left\{|R(u, v)|^{2}>\sigma^{2} \sqrt{2 \log (K N)}\right\}} .
$$

Note that the metric 2.6 allows for a blind search of pulses, when the DM values of pulses is unknown. In our analysis in Ch. 3 and Ch. 4, we will not use this option, since the DM value of all analyzed RRAT series is known.

The computational complexity of ETD is dominated by the term maxval $\times N \log (N)$, which is comparable to the complexity of the algorithm recently published by Zackay [19].

\subsubsection{Decision Rule}

Given a sequence of time-of-arrivals (TOAs) of potential pulses in a RRAT series, we crop chunks of the spectrogram around each time-of-arrival (TOA), then apply SFT to each chunk and calculate its ETD. The exact process of partitioning data into chunks is described in Ch. 3.

To have a rule which allows us to differentiate candidate pulses from noise, we select 100 chunks of noise from the same RRAT series. Chunks containing noise only are collected from the space between the chunks with potential pulses. These chunks are transformed to the SFT space and an ETD value for each chunk is found.

To make a distinction between pulses and noise, among the values of ETD of noise we picked the maximum value of ETD and set it as a threshold in both $16 \times 16$ and $32 \times 32$ areas. Mathematically it is described as

$$
d_{\text {maxnoise }}(\text { maxval })=\max _{(i \in(1, \ldots, N))} d_{i}(\text { maxval })
$$

If $d_{\text {candidate }}($ maxval $=16)>d_{\text {maxnoise }}($ maxval $=16)$

and $d_{\text {candidate }}($ maxval $=32)>d_{\text {maxnoise }}($ maxval $=32)$, then we claim that the candidate is a pulse. Otherwise, we decide that the candidate is noise.

\subsection{Algorithm 2: Matched Filtering in SFT domain (MF-SFT)}

As an alternative to Algorithms 1 and 3 described in this chapter, we suggest to apply a twodimensional matched filter approach described in details in a recently accepted for publication 
MNRAS paper [20]. It requires (1) develop a bank of two-dimensional matched filters with the filter response mimicking an underlying pulse in a spectrogram. Since the true shape and width of the pulse are unknown, we would have to develop a number of filters parameterized by width and DM values of the pulse; (2) each filter is spatially correlated with the tested spectrogram resulting in a two-dimensional correlation function; (3) peak signal-to-noise ratio ( $\overline{\mathrm{PSNR}}$ ), a performance metric frequently used in image restoration and enhancement [16], used as a decision metric; (4) the bank of matched filters are also spatially correlated to a set of spectrograms containing noise only; (5) a decision rule that relates the maximum value of PSNR of the tested spectrogram and the PSNR values of the spectrograms containing noise only is applied.

\subsubsection{Implementation of Matched Filters in the SFT Domain}

From the theory of the Fourier transform, spatial correlation in a two-dimensional space is a computationally expensive signal processing operation. Its $\mathbf{S F T}$ counterpart is much more efficient compared to the spatial correlation. From the convolution (correlation) property of the SFT, spatial frequencies of a test spectrogram and of a matched filter simply multiply.

Let $\gamma(t, f: D M, W)$ be a spectrogram of a simulated pulse parameterized by a $\overline{\mathrm{DM}}$ value and a pulse width $\mathrm{W}$ (for details of how to simulate dispersed pulses see 2.4). No noise is added to the pulse. Let $\Gamma(u, v: D M, W)$ be its two-dimensional Fourier transform. As before, denote a test spectrogram transformed to the SFT domain as $R(u, v)$. Similar to the case of Algorithm 1, to match each filter to the noisy transformed data, we zoom on a small area around the zero frequency and multiply $R(u, v)$ by the complex conjugate of each response $\Gamma(u, v: D M, W)^{*}$

\subsubsection{Decision Metric}

Since the product of $R(u, v)$ and $\Gamma(u, v: D M, W)^{*}$ is complex valued in general, we use the energy in correlated data (ECD) as a performance metric:

$d_{E C D}(D M, W)=\sum_{(u, v): 0<u, v<\text { maxval }}^{\infty}\left|R(u, v) \Gamma^{*}(u, v: D M, W)\right|^{2} \times \Phi_{\left(\left|R(u, v) \Gamma^{*}(u, v: D M, W)\right|^{2}>\sigma^{2} \sqrt{2 \log (K M)}\right)}$,

where we used the same rule of selection of informative pixels as before. 

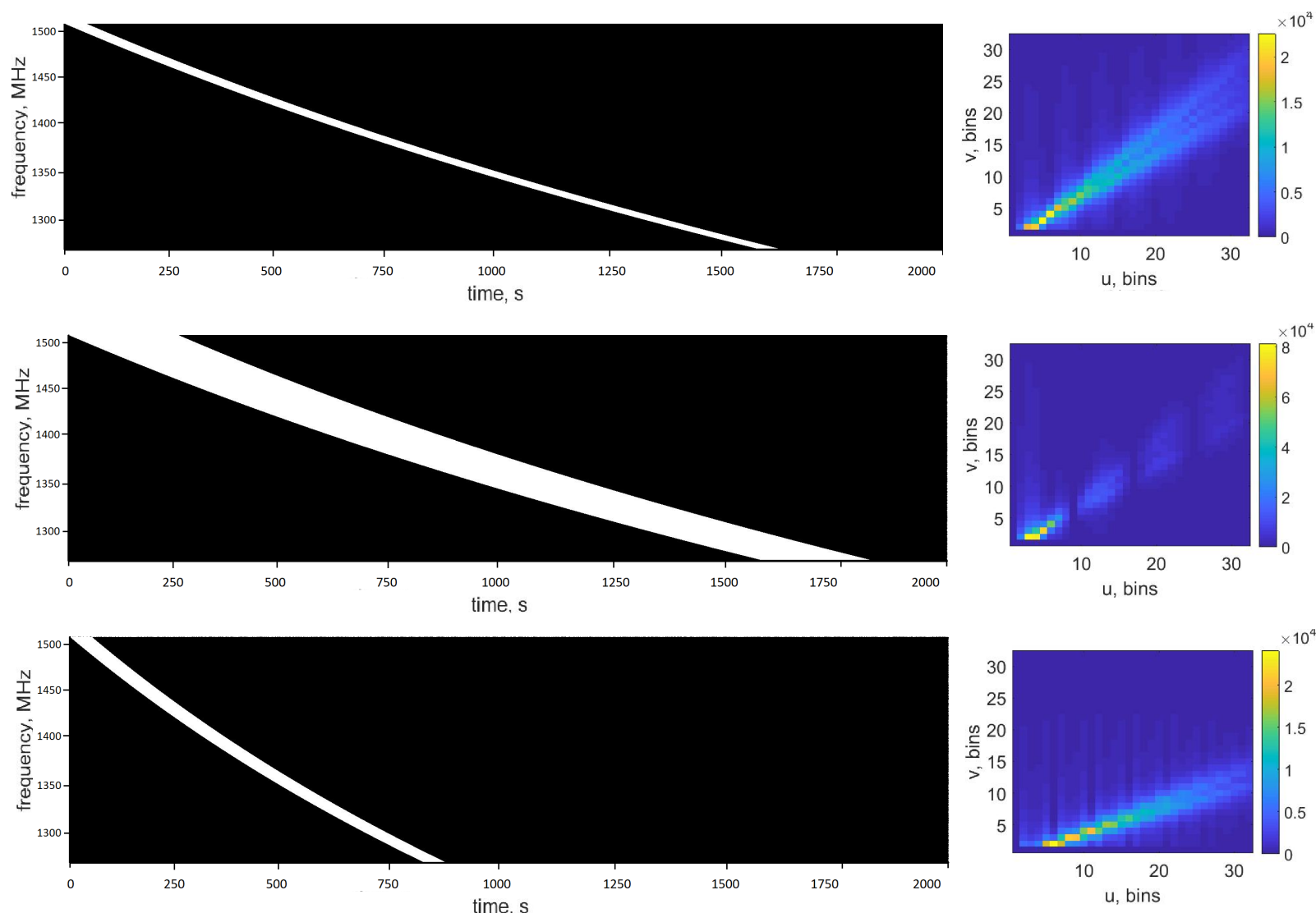

Figure 2.4: The left panels show spectrograms of three noise free dispersed pulses. The right panels show their three SFT signatures. The first row presents a pulse parameterized by $D M=$ $196 \mathrm{pc} / \mathrm{cm}^{3}$ and $\mathrm{W}=5 \mathrm{~ms}$. The second row presents a pulse parameterized by $D M=196 \mathrm{pc} / \mathrm{cm}^{3}$ and $\mathrm{W}=25 \mathrm{~ms}$. The last row is a pulse generated with $D M=100 \mathrm{pc} / \mathrm{cm}^{3}$ and $\mathrm{W}=5 \mathrm{~ms}$.

Figure 2.5 shows an example of the SFT signature of a noisy spectrogram and the SFT signature of a dispersed pulse. The generated dispersed pulse plays the role of spatial filter. The third figure shows the energy of the product of the two functions.

\subsubsection{Decision Rule}

To distinguish candidate pulses from noise, we identify a set of spectrograms containing noise only, extract the dependence of the $\mathrm{ECD}$ as a function of $\overline{\mathrm{DM}}$ value from each spectrogram and then find the maximum ECD value as a function of DM. Mathematically, this step can be described as follows. Let $d_{1}(D M), \ldots, d_{L}(D M)$ be $L E C D$ functions extracted from $L$ spectrograms of noise. 


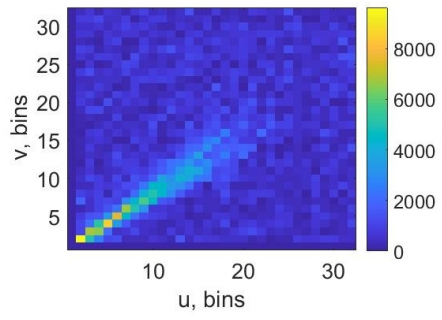

(a) SFT of noisy pulse

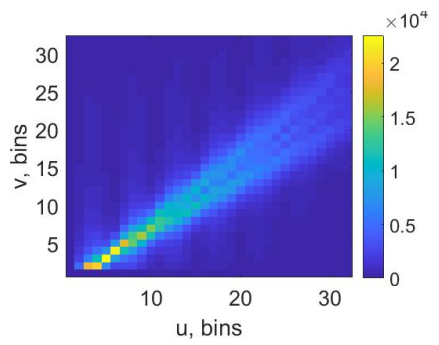

(b) SFT of dispersed pulse

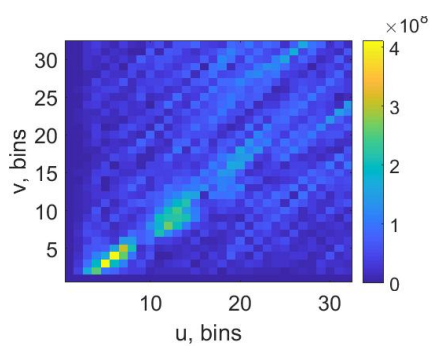

(c) Energy of (a) and (b)

Figure 2.5: The left panel shows the SFT signature of a noisy pulse of J1819-1458. The second panel shows a simulated SFT signature of a dispersed pulse with the same DM and width of J1819-1458, which is used as mask. The third figure shows the energy of the product of the two signatures.

The maximum over the $L$ functions for a given DM value is

$$
d_{\text {max noise }}(D M)=\max _{i \in\{1, \ldots, L\}} d_{i}(D M)
$$

To decide if raw data contain a pulse, we form a ratio of $d_{\text {raw data }}(D M)$ to $d_{\text {max noise }}(D M)$, then search for a peak. Then the detection rule is based on the maximum value of the ratio:

$$
\text { Pulse is detected, if } \max _{D M}\left\{\frac{d_{\text {raw data }}(D M)}{d_{\text {max noise }}(D M)}\right\} \geq 1 .
$$

Pulse is not detected, otherwise. This is an equivalent of determining the probability of detection of the algorithm at an empirical zero false alarm rate. We will use the probability of detection to characterize the performance of the SFT based method.

Note we neglected the dependence of the metric on the pulse width W. In the SFT domain, $\mathrm{W}$ affects the length of the "fan" that we observe. We found that fixing it at $\mathrm{W}=5 \mathrm{~ms}$ generates sufficiently good results in terms of the detection performance of Algorithm 2. This parameter can be varied if needed.

\subsection{Algorithm 3: Conventional Algorithm (CA)}

The algorithm that we call here Conventional Algorithm $(\overline{\mathrm{CA}})$ is the one which is used by most of radio astronomers for the search of isolated astrophysical pulses.

The conventional algorithm can be described in a few steps: 
- Convert the raw data collected at the back end of a radio telescope into a filterbank format.

- Dedisperse the filterbank data at the $\overline{\mathrm{DM}}$ of each $\mathrm{RRAT}$ and also at a zero DM by using the software named SIGPROC [21]. Since high frequencies of pulses travel faster through the ISM compared to lower frequencies, the lower frequency components of the pulse are observed by the receiver $\Delta t$ seconds later compared to high frequency components. This phenomenon is known as dispersion. To dedisperse the data, we shift data in each frequency channel by the amount of time equal to the delay experienced by the frequency component of the pulse and thus align pulse components across all channels into a vertical column. To improve the $\widehat{S N R}$ of the pulse the pulse components are integrated across all frequency channels resulting in a one dimensional signal displayed as a function of time. An example of a spectrogram and a one dimensional signal after its dedispersion is shown in Fig. 2.6.

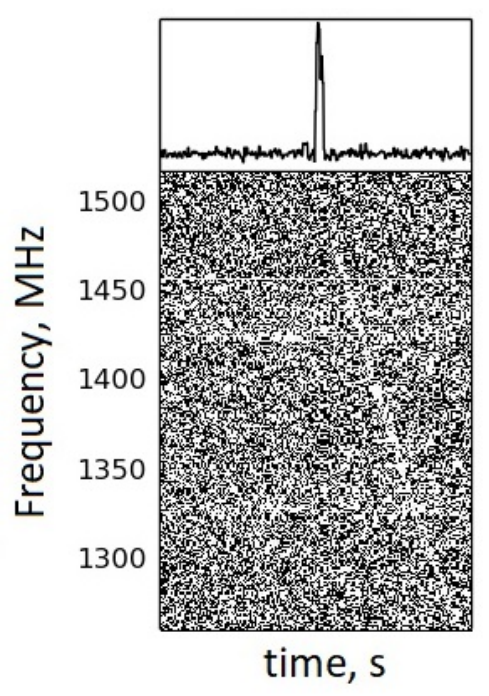

Figure 2.6: Shown is a bright pulse of J1819-1458. The lower panel displays a spectrogram of a dispersed pulse. The upper panel displays its time series after dedispersion.

- Apply a matched filter with a Gaussian profile to the dedispersed time series and search for pulses exceeding in value a $5 \sigma$ threshold. Here $\sigma$ is the noise standard deviation after the series is matched filtered. Pulses with $\underline{\mathrm{SNR}}$ values above $5 \sigma$ are considered to be candidate pulses [22].

- Manually inspect the candidate pulses to verify their astrophysical nature. 


\section{Chapter 3}

\section{Data}

In this chapter we describe the data which we use to evaluate performance of the three algorithms presented in Chapter 2. We also discuss data preprocessing steps which we applied to the data prior to the detection algorithms. At the end of this chapter we discuss the timing solution used to locate potential pulses in each RRAT series.

\subsection{Data Used and Initial Data Processing}

In our analysis we used ten RRAT time series. All time series were acquired by the SCAMP datataking machine at the Parkes Telescope. The data has 512 frequency channels. The total bandwidth of the Parkes' receiver is $256 \mathrm{MHz}$. The highest frequency of the receiver band was set to $1518 \mathrm{MHz}$. The lower frequency of the band was set to $1262 \mathrm{MHz}$. All data were stored in a binary form, with 1 bit per sample. The temporal length of the analyzed times series varied from 20 minutes to 30 and 60 minutes. The sampling interval in filterbank data was set to $100 \mu \mathrm{s}$. The ten selected RRAT time series are listed in Table 3.1 together with the observational parameters describing every RRAT. The parameters of the conventional pulse detection algorithm applied to the data are listed in Table 3.2 .

Before applying the three detection algorithms to the RRAT time series, we performed a number preprocessing steps. They are as follows:

- We first transformed raw time series with the extension .dat (data files) into (.dat.fil) filterbank data format by using program FILTERBANK [21] on bowser, a server located in 
Physics and Astronomy Department at WVU.

- As a next step we saved a single .dat.fil file into 16 .ascii files parameterized by different frequency ranges divided into groups of frequency channels (1 to 32,33 to 64,65 to 96 , 97 to 128,129 to 160,161 to 192,193 to 224,225 to 256,257 to 288,289 to 320,321 to 352,353 to 384,385 to 416,417 to 448,449 to 480 , and 481 to 512 frequency channels) by using program READER [21] on bowser, since we implemented new detection algorithms in MATLAB which can not read dat.fil files, or the whole array as large as time series data. While saving data into different frequency ranges, a column of sample time was automatically saved to the first column so that there were 33 columns per each ascii file.

- As a following step, 16 .ascii files are transformed into 16 .mat files by using function textread in MATLAB. Simultaneously, we removed the first column in every .ascii file, which represents the sampling time. After this we adjusted the time series of all frequency channels (the channels 1 through 8 had a different length from all other channels) to the same length. For example, if a file has an observation time of 60 minutes, it would have a length of 36,000,000 samples. However the first eight frequency channels have the length of $36,000,001$.

- We calculated the time delay $\triangle t$ of pulses in a RRAT time series and made a decision about the temporal size of the window into which we are going to partition each time series. This step is needed to apply the two proposed algorithms to RRAT data.

$$
\triangle t=4.149 \times 10^{3} \times D M \times\left(\frac{1}{f_{\text {low }}^{2}}-\frac{1}{f_{\text {high }}^{2}}\right)
$$

Based on our calculations, we chose windows of six different lengths. A window can be set to the length of $0.05,0.1,0.15,0.2,0.25$ and $0.3 \mathrm{sec}$. For example, J1819-1458 has a $\triangle t$ of $0.1577 \mathrm{sec}$. To ensure that the entire pulse is displayed in a window, but no additional noise is included in it, we set the window to the size of $0.2 \mathrm{sec}$. All window sizes selected for performance testing are listed in Table 3.1 .

- To analyze each pulse, we selected 7 different windows with a shift of $1 / 5$ of the length of the window around the anticipated location of each potential pulse. The overlap is set to be 


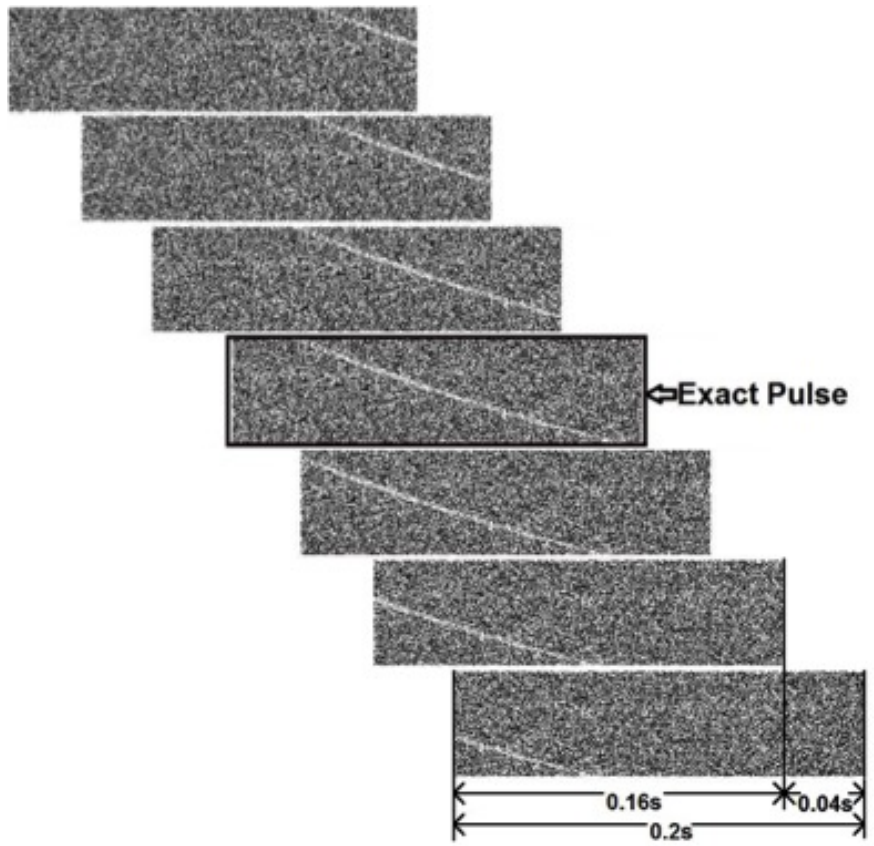

Figure 3.1: Choosing the size of the window for J1819-1458. Shown are seven windows. Each following window is shifted $0.04 \mathrm{sec}$ to the right or left of the previous window.

$4 / 5$ of the window length. In this case, we make sure that one of the seven windows contains the entire pulse. Figure 3.1 illustrates a window of length $0.2 \mathrm{sec}$, and its shifted versions with an overlap of $0.16 \mathrm{sec}$ for J1819-1458.

\subsection{Timing Solution}

RRATs are irregularly emitting pulsars. If they would be normal pulsars they would emit regularly, every rotating period. However, not every pulse can be observed in a RRAT series. To

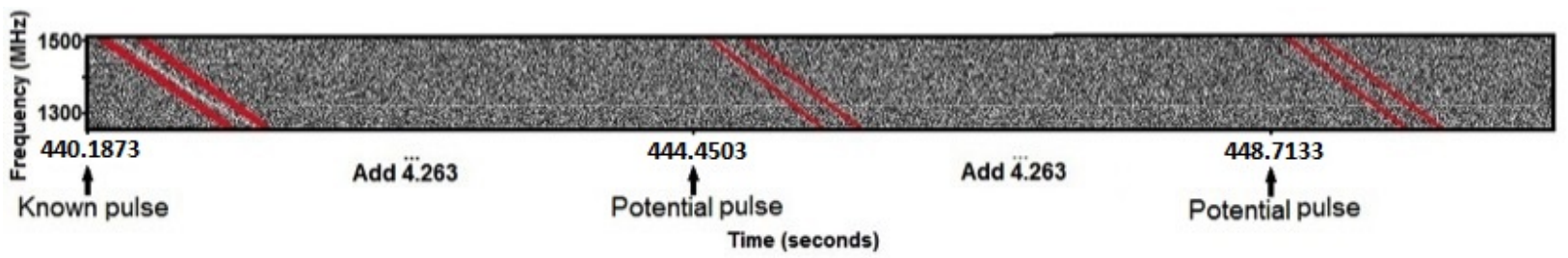

Figure 3.2: Calculate TOA of potential pulses by adding $n \times$ Period to the TOA of a known pulse. For J1819-1458, a strong known pulse is located at $440.1873 \mathrm{sec}$. The period of this RRAT is $4.263 \mathrm{sec}$. By adding and subtracting one period at a time, we obtained the TOAs of potential pulses. Thus pulses detected at the locations $444.4503 \mathrm{sec}, 448.7133 \mathrm{sec}$ and so on. 
save processing time we choose to work with windows of potential pulses. To be more specific, since we know the period of every RRAT, we form windows around a few bright pulses first. To calculate the TOA of other potential pulses, we selected one brightest pulse from each data file as a known pulse, and then added or subtracted one period, two periods, and so on. The brightest pulses of each data file are listed in Table 3.1. After identifying the location of each potential pulse in a RRAT series, we formed the seven shifted windows around the TOA of every potential pulse in hope that one of the windows will contain the entire potential pulse. We illustrate this process on the time series of J1819-1458 as shown in Figure 3.2 


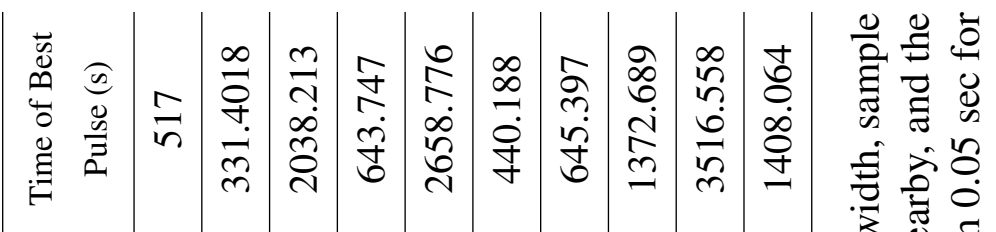

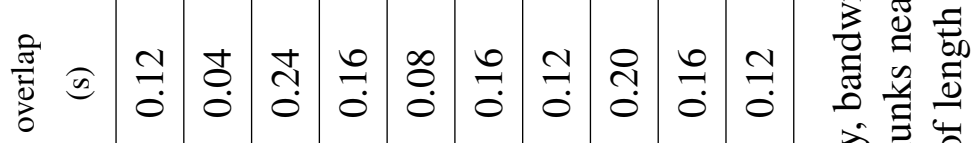

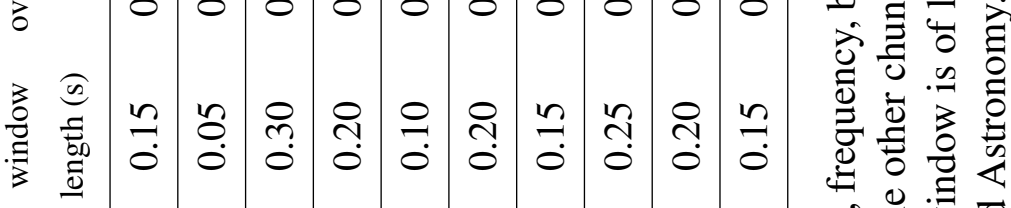

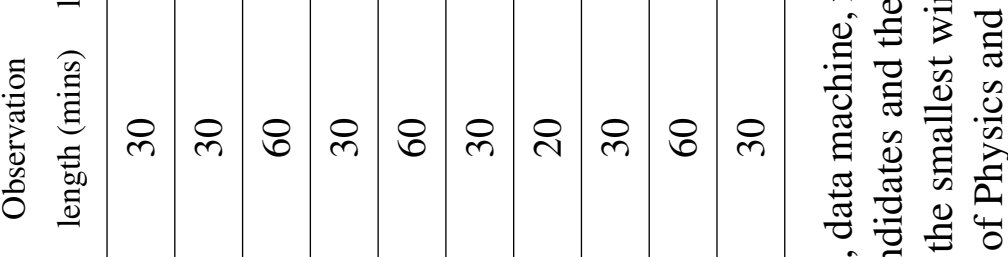

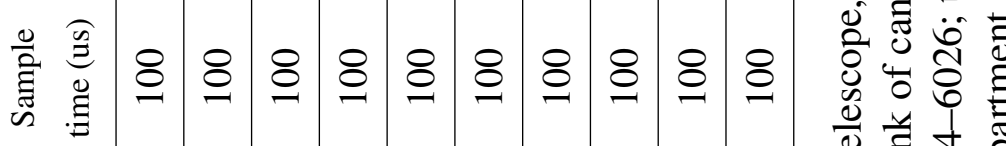

$$
\begin{aligned}
& \text { 言 }
\end{aligned}
$$

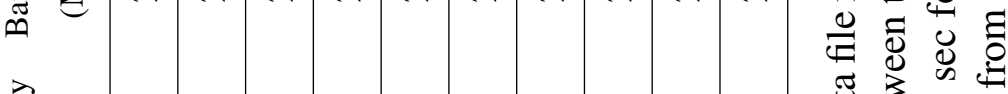

$$
\begin{aligned}
& \text { 离 }
\end{aligned}
$$

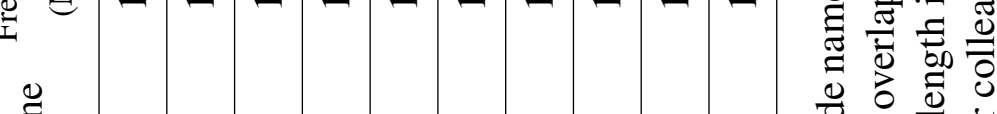

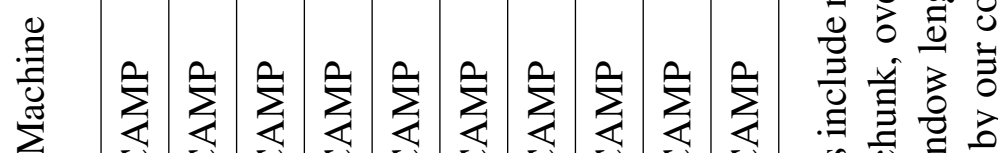

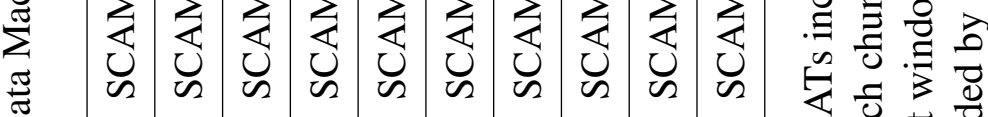

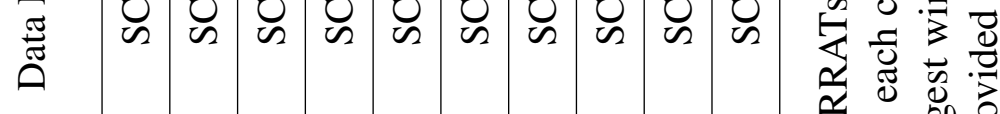

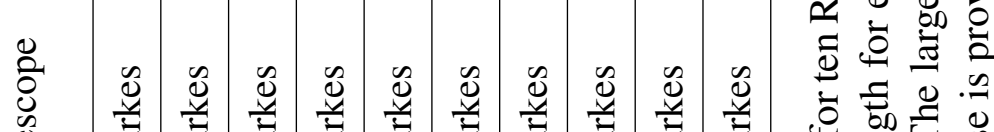

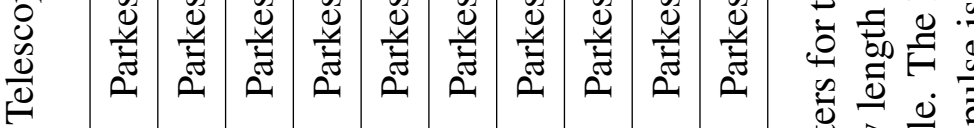

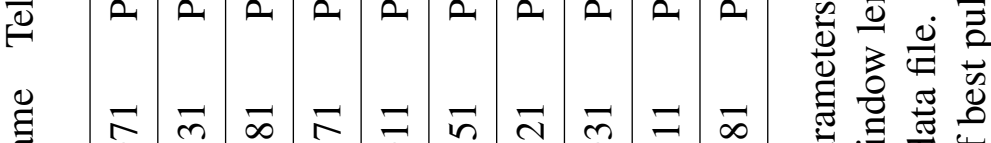

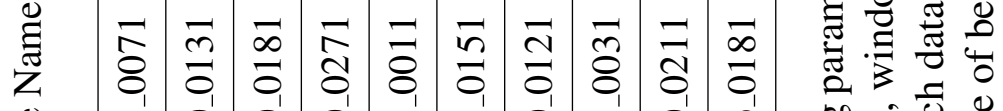

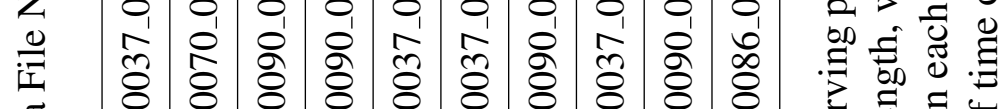

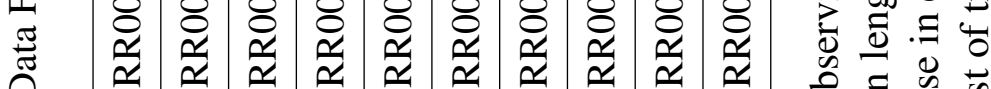

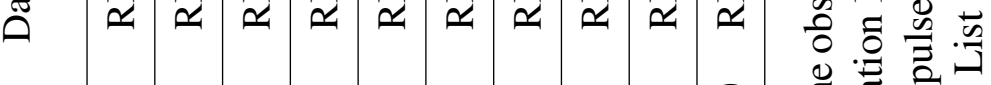

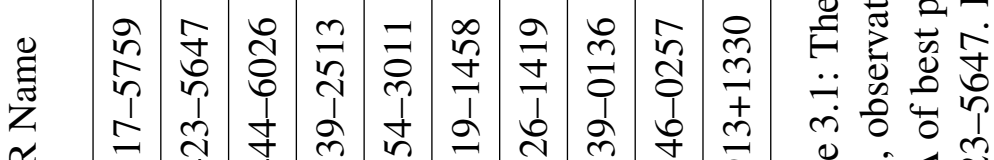

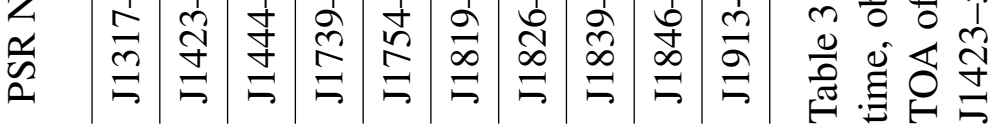




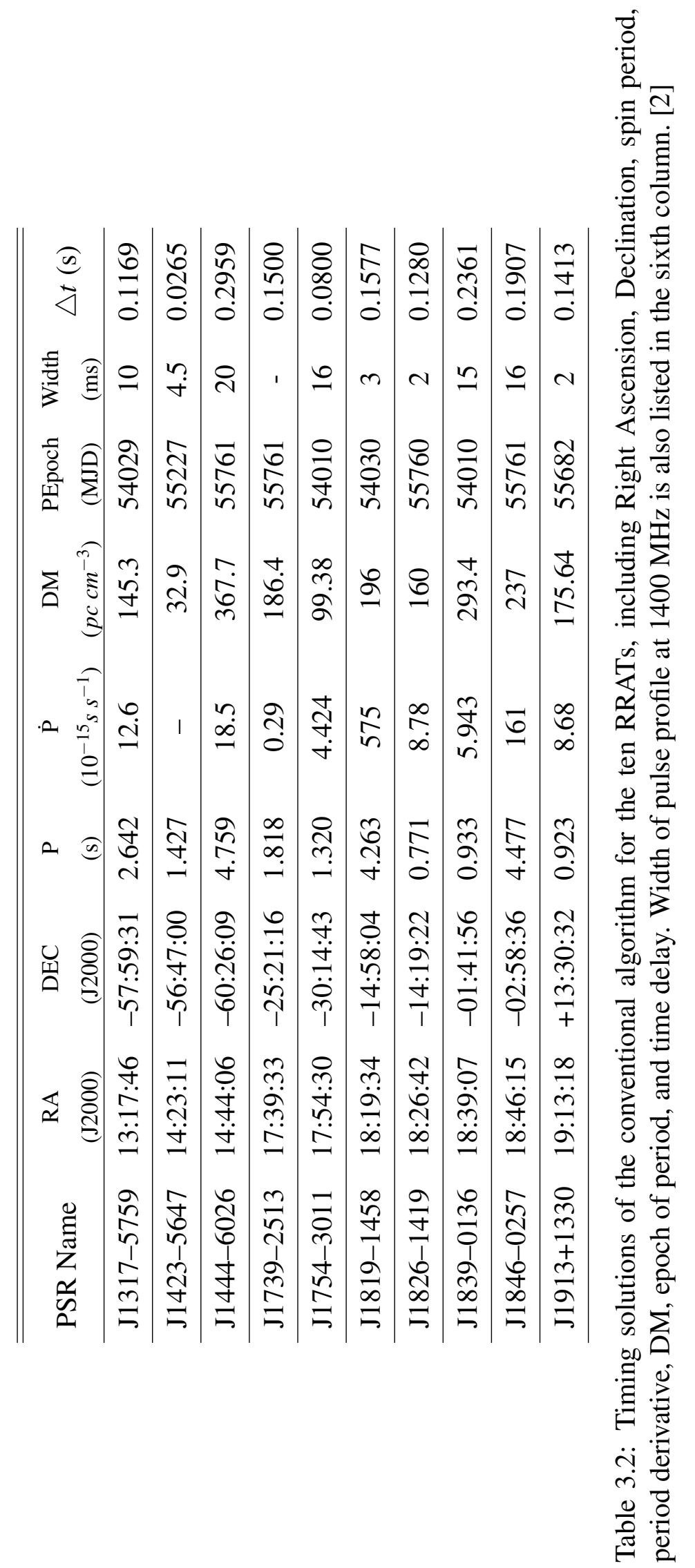




\section{Chapter 4}

\section{Experimental Results}

The pulse detection rules developed and described in the previous chapters were tested on filterbank data of ten different RRATs,

\subsection{Performance Comparison}

The performance of the three algorithms is summarized in Tables 4.74 .16 . The Energy in Transformed Data (ETD) and the Conventional Algorithms (CA ) were applied to every location of a potential pulse. To confirm pulse candidates detected by ETD, we applied Matched Filtering in the Spatial Fourier Transform domain (MF-SFT) to the locations with pulses already detected by either of the other two methods. The number of candidate pulses for each RRAT series are shown in Table 4.1 .

The application of the conventional algorithm with the detection threshold set to $5 \sigma$ to the RRAT data results in a large number of candidate pulses. After inspecting the candidates, pulses due to $\overline{R F I}$ and spikes caused by noise are manually removed. The false alarm rate (FAR $)$ of the conventional algorithm for the threshold of $5 \sigma$ is shown in Table 4.2 .

There are three major steps of manual inspection. First, a pulse is a candidate if its value exceeds $5 \sigma$ and is at the right phase. According to the periodicity of RRATs, pulses can be observed only at certain locations. If a peak is not located at the expected phase, it is most likely noise or RFI. Second, a candidate pulse is smoothed by using rebinning in time. The smoothing level should not be too large or too small. In most cases selecting it between 4 and 7 is ideal. Re-binning 


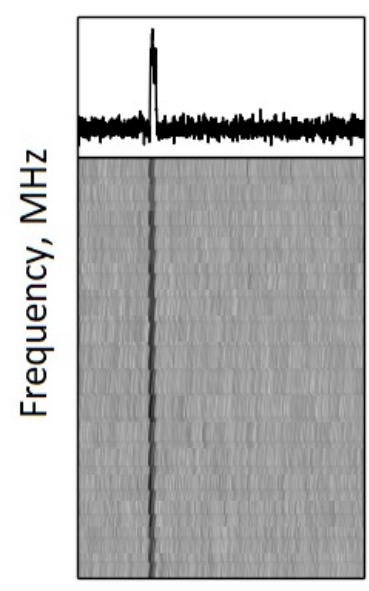

time, s

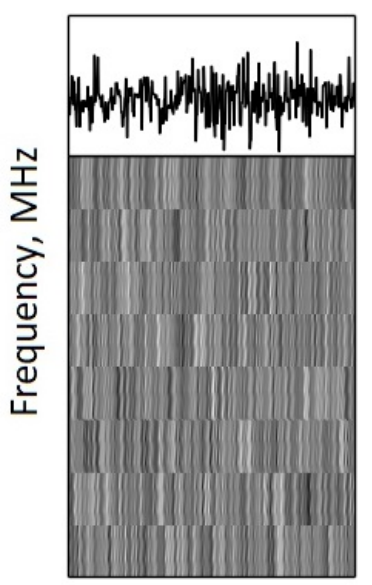

time, $s$

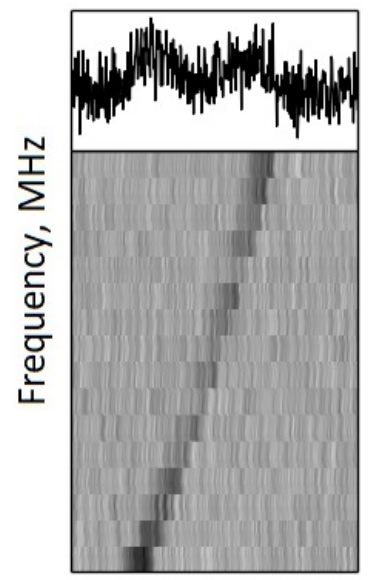

time, s

Figure 4.1: Shown are several candidates detected by the conventional algorithm. The left panel shows an example of a composite pulse. The pulse can be seen as a vertical line in the dedispersed data on the left panel. The middle panel shows an example of noise. The right panel shows an example of RFI, which can be seen as an approximately diagonal line in dedispersed data.

is a process of adding up the adjacent bins of data in an attempt to decrease the level of noise in the data, which also improves $\mathrm{SNR}$ of the pulse. The re-binning level is the number of times the data are re-binned in time. If raw data have 1,000 time samples and are re-binned once (a level one re-binning is applied), then there will be 500 columns in a new window. Third, after reducing the resolution of a dedispersed time series (now performed in frequency direction) a candidate dedispersed pulse is announced as a detected pulse only if the pulse is visible as a vertical line in frequency channels.

Examples of pulses manually classified as RFI and of detected astrophysical pulses are shown in Figure 4.1 Figure 4.2, Figure 4.3, and Figure 4.4 show three examples of different smoothing levels of a bright pulse, a weak pulse and noise.

We employ the conventional algorithm as a baseline for our analysis. Using the pulses detected by this algorithm as the ground truth we present the detection rates of the other two algorithms, ETD and MF-SFT in Table 4.3.

When the conventional algorithm does not detect a candidate pulse but the ETD algorithm suggests that this is a candidate pulse, we do not have a means to confirm that the pulse detected by the ETD is a real pulse. In this case, we apply the algorithm performing MF-SFT to have a third "opinion" about the presence or absence of a pulse. It is interesting to note that the total percentage 

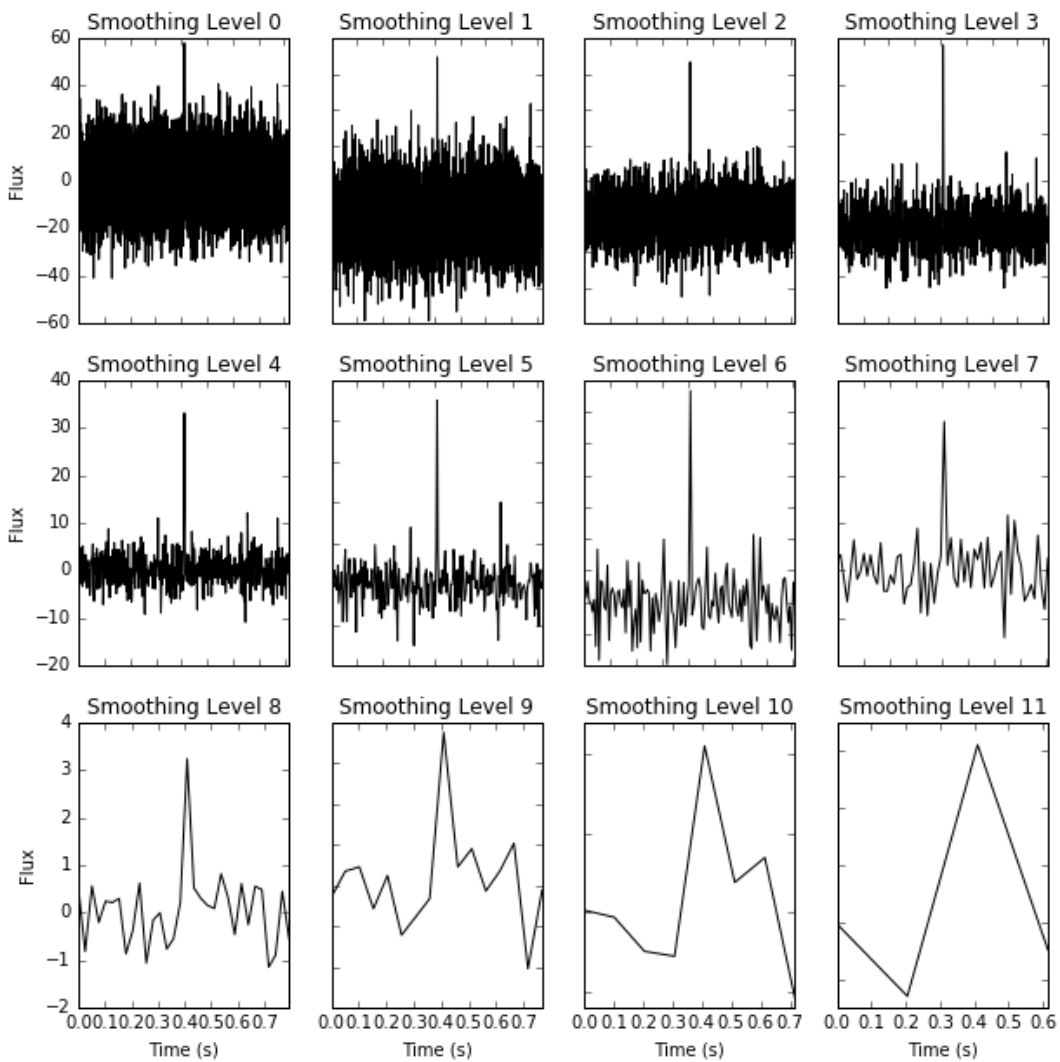

Figure 4.2: This figure shows different smoothing levels of a bright pulse. Smoothing level 0 means the data is not smoothed. We can see that this bright pulse is visible even when it is not smoothed. When the smoothing level is larger, the pulse is more visible and has a higher SNR. 

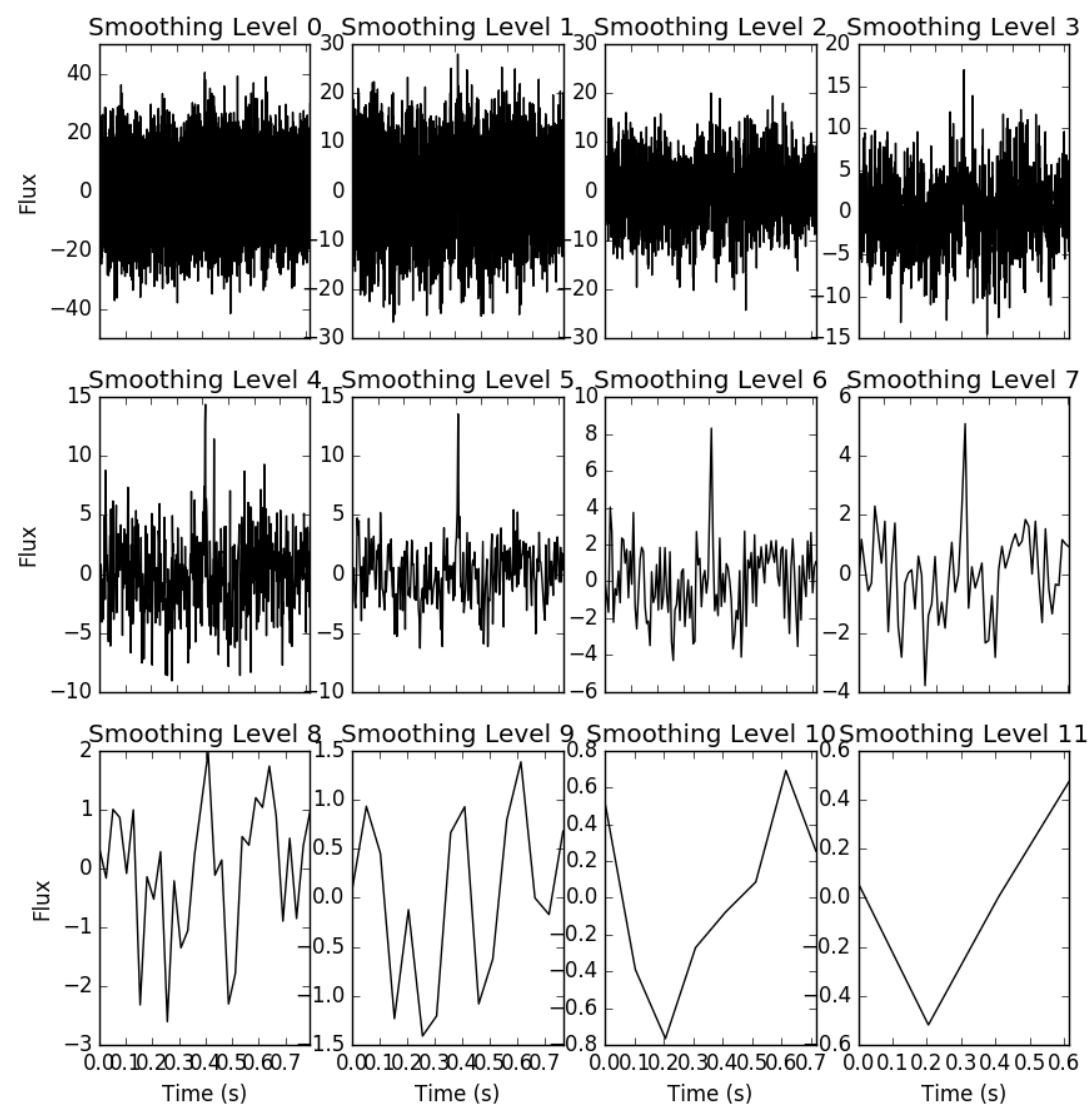

Figure 4.3: This figure shows different smoothing levels of a weak pulse. The peak of pulse is not significant, when smoothing level is 0 to 2 . When the smoothing level is larger, the pulse is more significant. 

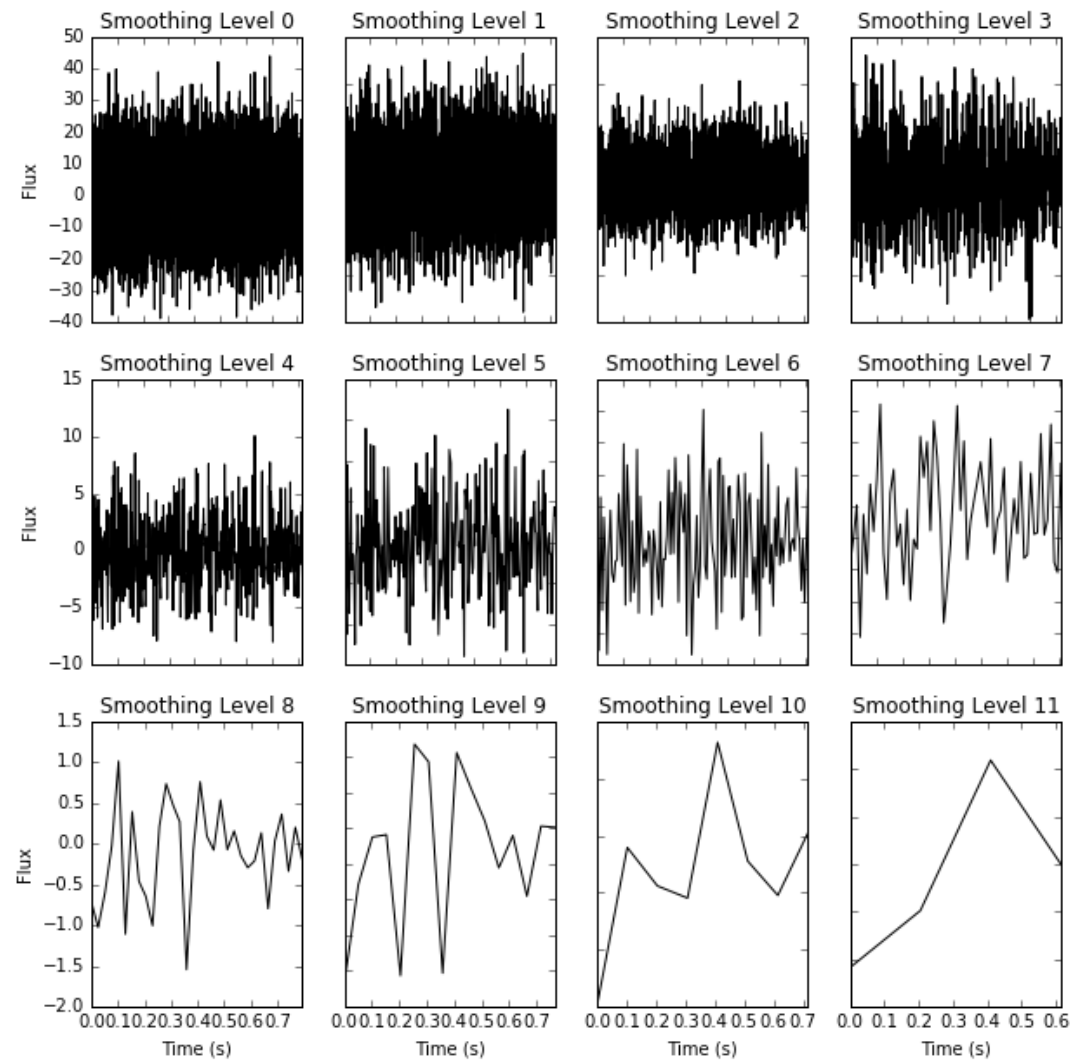

Figure 4.4: This figure shows different smoothing levels of white noise. There is no significant peak of pulse showing up from smoothing level 1 to 9. 


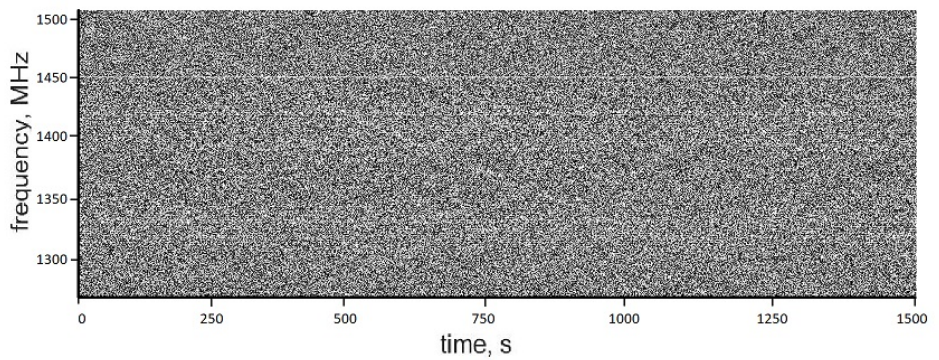

(a)

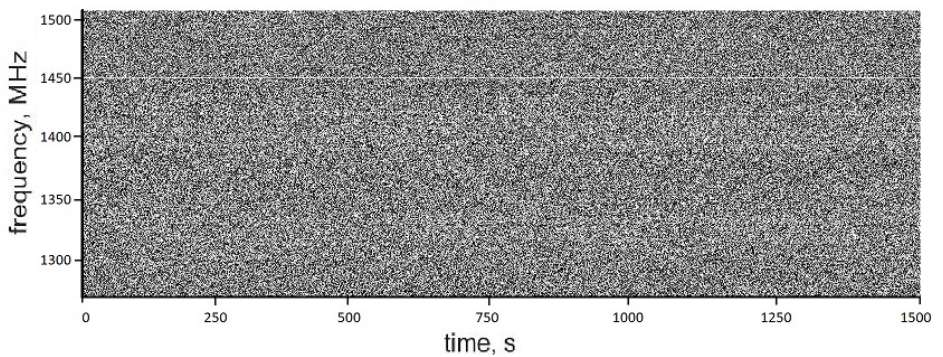

(c)

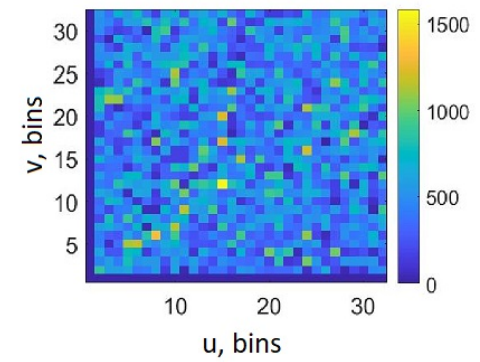

(b)

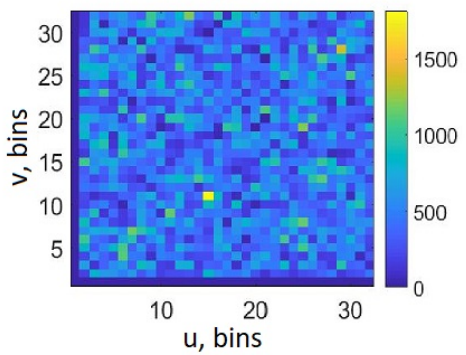

(d)

Figure 4.5: (a) Spectrogram of the pulse with the TOA at 645.3976 sec. in RR0090_0121 and SNR of 16.1. The pulse is detected by both the conventional algorithm and by the ETD algorithm. (b) Signature of the pulse in (a) in the SFT domain. (c) Spectrogram of the pulse at $661.5901 \mathrm{sec}$. in RR0090_0121 with SNR of 4.1. The pulse is detected by the ETD algorithm but not detected by the conventional algorithm. (d) The signature of this pulse in the SFT domain.

of candidate pulses detected by the ETD algorithm and confirmed by MF-SFT, but not detected by the conventional algorithm, is equal to $79.1 \%$.

Two candidate pulses from the RRAT time series RR0090_0121 of J1826-1419 are shown in Figure 4.5. The top pulse with the conventional SNR of 10.5, is detected both by the conventional algorithm and by ETD. The pulse at the bottom, with the SNR value of 3.4, is detected by the ETD algorithm, but not detected by the conventional algorithm with the detection threshold set to $5 \sigma$. Note that the two pulses are very faint, but can be seen by a trained eye, both in time series and the SFT domain. They have the same $\overline{\mathrm{DM}}$ value (based on the slope of the dispersed pulses) and a very similar signature in the SFT domain. Two other examples from the RRAT time series RR0086_0181 of J1913+1330 and the RRAT time series RR0037_0071 of J1317-5759 are shown in Figure 4.6 and Figure 4.7 . 


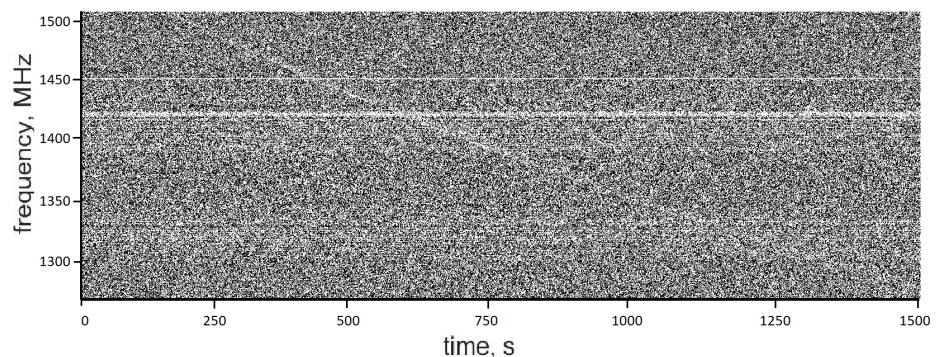

(a)

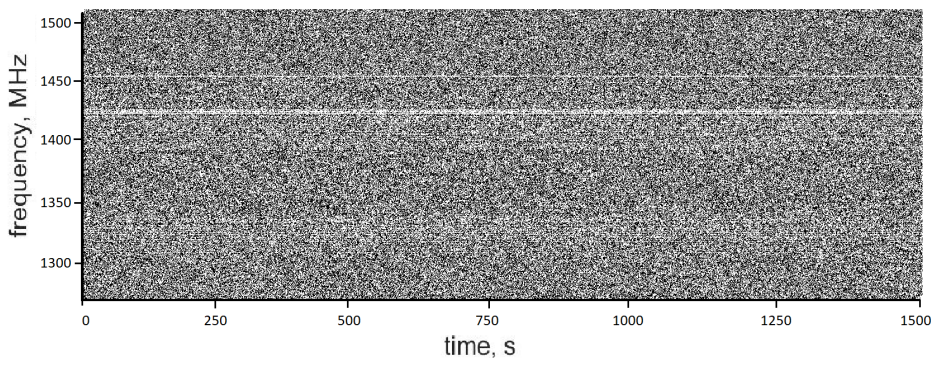

(c)

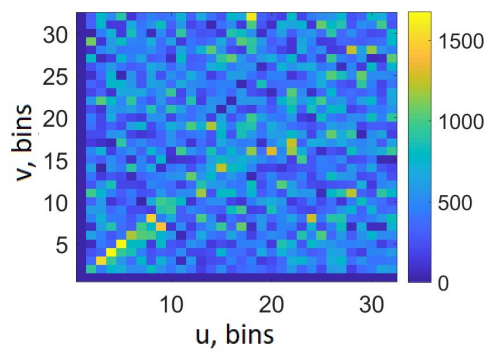

(b)

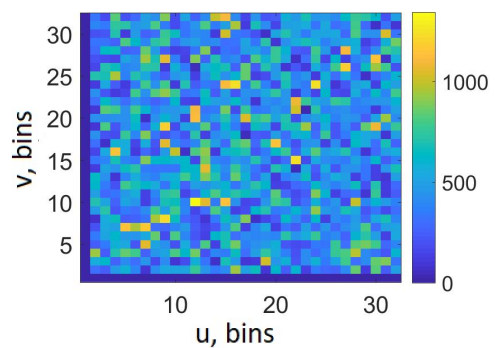

(d)

Figure 4.6: (a) Spectrogram of the pulse at 1408.0501 sec. in RR0086_0181 and SNR of 19.6. The pulse is detected by both the conventional algorithm and by the ETD algorithm. (b) Signature of this pulse in the SFT domain. (c) Spectrogram of the pulse at $1757.9401 \mathrm{sec}$. in RR0086_0181 with SNR of 2.8. The pulse is detected by the ETD algorithm but not detected by the conventional algorithm. (d) Signature of this pulse in the SFT domain. 


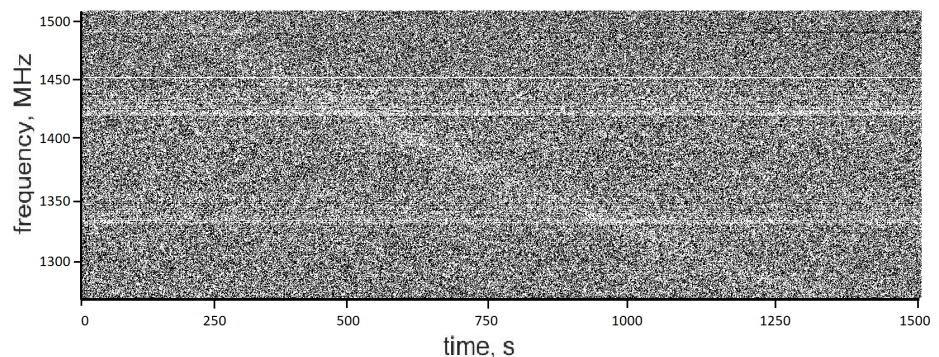

(a)

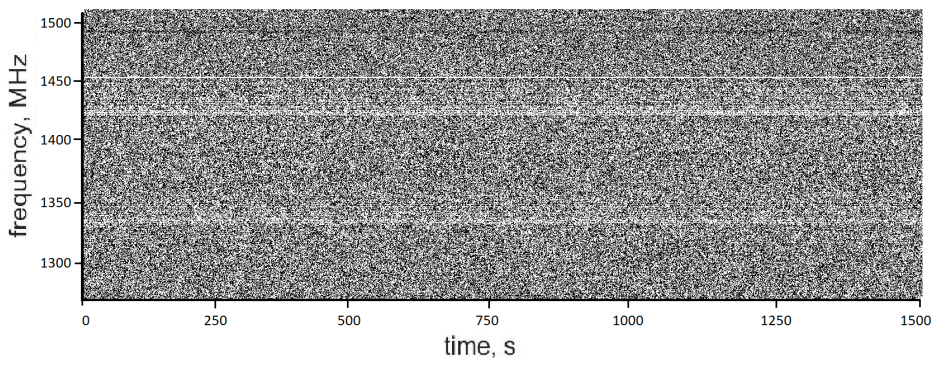

(c)

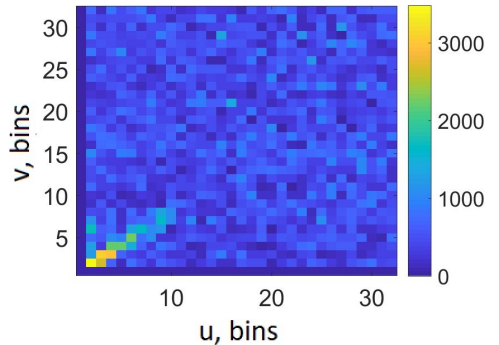

(b)

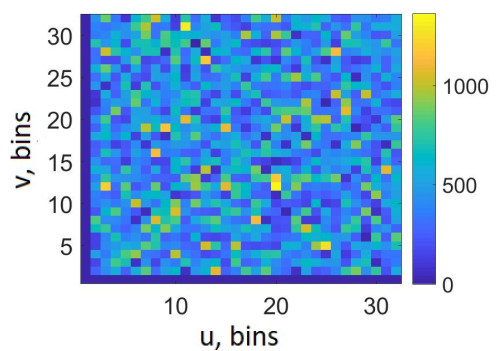

(d)

Figure 4.7: (a) Shown is a spectrogram of the pulse timed at 516.9901 sec. in RR0037_0071. The pulse is detected by both the conventional algorithm and by the ETD algorithm. (b) The signature of this pulse in the SFT domain. (c) Shown is a spectrogram of the pulse at $305.7601 \mathrm{sec}$. in RR0037_0071. The pulse is detected by the ETD algorithm but not detected by the conventional algorithm. (d) The signature of this pulse in the SFT domain. 


\begin{tabular}{|c|c|c|c|c|c|c|}
\hline Data File Name & PSR Name & $\begin{array}{c}\text { Detected } \\
\text { by CA }\end{array}$ & $\begin{array}{c}\text { Detected } \\
\text { by ETD }\end{array}$ & $\begin{array}{c}\text { Detected } \\
\text { by MF-SFT }\end{array}$ & $\begin{array}{c}\text { Detected by } \\
\text { CA and ETD }\end{array}$ & $\begin{array}{c}\text { Detected by } \\
\text { ETD and MF-SFT }\end{array}$ \\
\hline \hline RR0037_0011 & $\mathrm{J} 1754-3011$ & 6 & 8 & 7 & 0 & 4 \\
\hline RR0037_0031 & $\mathrm{J} 1839-0136$ & 1 & 30 & 30 & 0 & 29 \\
\hline RR0037_0071 & $\mathrm{J} 1317-5759$ & 13 & 41 & 25 & 7 & 21 \\
\hline RR0037_0151 & $\mathrm{J} 1819-1458$ & 48 & 30 & 50 & 27 & 30 \\
\hline RR0070_0131 & $\mathrm{J} 1423-5647$ & 1 & 2 & 2 & 1 & 2 \\
\hline RR0086_0181 & $\mathrm{J} 1913+1330$ & 7 & 5 & 9 & 2 & 5 \\
\hline RR0090_0121 & $\mathrm{J} 1826-1419$ & 16 & 23 & 28 & 4 & 18 \\
\hline RR0090_0181 & $\mathrm{J} 1444-6026$ & 2 & 1 & 2 & 1 & 1 \\
\hline RR0090_0211 & $\mathrm{J} 1846-0257$ & 7 & 1 & 6 & 0 & 1 \\
\hline RR0090_0271 & $\mathrm{J} 1739-2513$ & 3 & 12 & 13 & 0 & 10 \\
\hline Total & - & 104 & 153 & 172 & 42 & 121 \\
\hline
\end{tabular}

Table 4.1: The second, third and forth columns present the number of candidate pulses detected by each individual algorithm. The fifth column lists the number of candidate pulses detected by both the conventional algorithm and by the ETD algorithm. The sixth column is the number of candidate pulses detected by both the ETD algorithm and by the algorithm performing Matched Filtering in the SFT domain. 


\begin{tabular}{|c|c|c|c|c|}
\hline File Name & Number of Candidates & Detections & Non-candidates & False Alarm Rate \\
\hline \hline RR0037_0011 & 6 & 82 & 76 & $92.7 \%$ \\
\hline RR0037_0031 & 1 & 9 & 8 & $88.9 \%$ \\
\hline RR0037_0071 & 13 & 24 & 11 & $45.8 \%$ \\
\hline RR0037_0151 & 48 & 73 & 25 & $34.2 \%$ \\
\hline RR0070_0131 & 1 & 14 & 13 & $92.9 \%$ \\
\hline RR0086_0181 & 7 & 27 & 20 & $74.1 \%$ \\
\hline RR0090_0121 & 16 & 25 & 9 & $36.0 \%$ \\
\hline RR0090_0181 & 2 & 29 & 27 & $93.1 \%$ \\
\hline RR0090_0211 & 7 & 28 & 21 & $75.0 \%$ \\
\hline RR0090_0271 & 3 & 22 & 19 & $86.4 \%$ \\
\hline Total & 104 & 333 & 229 & $68.8 \%$ \\
\hline
\end{tabular}

Table 4.2: The second column is the number of candidate pulses detected by the conventional algorithm after manual inspection. It means they are confirmed pulses. The third column presents the number of candidate pulses detected by the algorithms but not inspected by eye. The fourth column lists the number of candidate pulses which are not confirmed after being inspected by eye. They are considered as RFI and pulses due to noise. The fifth column presents the false alarm rate of the conventional algorithm for each data file. The last row of the table shows the total number of candidate pulses in the second through the fourth columns and the average false alarm rate. 


\begin{tabular}{|l|l|l|l|l|l|l|}
\hline Algorithm & SNR $>10$ & SNR $>9$ & SNR $>8$ & SNR $>7$ & SNR $>6$ & SNR $>5$ \\
\hline \hline Conventional Algorithm & 27 & 37 & 41 & 51 & 68 & 104 \\
\hline $\begin{array}{l}\text { Energy in Transformed Data } \\
\text { Algorithm }\end{array}$ & 25 & 33 & 35 & 39 & 40 & 42 \\
\hline $\begin{array}{l}\text { Matched Filtering in SFT do- } \\
\text { main Algorithm }\end{array}$ & 27 & 37 & 43 & 51 & 67 & 93 \\
\hline $\begin{array}{l}\text { Energy in Transformed Data } \\
\text { Algorithm/Conventional Al- } \\
\text { gorithm }\end{array}$ & $92.6 \%$ & $89.2 \%$ & $81.4 \%$ & $76.5 \%$ & $58.8 \%$ & $40.4 \%$ \\
\hline $\begin{array}{l}\text { Matched Filtering in SFT do- } \\
\text { main Algorithm/Conventional } \\
\text { Algorithm }\end{array}$ & $100.0 \%$ & $100.0 \%$ & $100.0 \%$ & $100.0 \%$ & $98.5 \%$ & $89.4 \%$ \\
\hline
\end{tabular}

Table 4.3: The second column is the number of candidate pulses detected by the conventional algorithm with SNR exceeding 10, 9, 8, 7, 6, and 5. The third and fourth columns show how many candidate pulses, which have been detected by the conventional algorithm, are also detected by the ETD algorithm and by the algorithm implementing Matched Filtering in the SFT domain, respectively. The fifth and sixth columns present the percentage of detected candidate pulses by the ETD algorithm and by the algorithm implementing Matched Filtering in the SFT domain. 


\subsection{SNR calculated by a Matched Filter with a Gaussian vs. Rectangular Profile}

To calculate the SNR value for each detected pulse, we involved two matched filters, one with a Gaussian profile and the other one with a rectangular profile. These filters were applied to dedispersed time series. These filters denoise data resulting in a more visible pulse and a smoothed noise after their application. The profile of a pulse is often modeled as a Gaussian profile. It also can be modeled as a Gaussian profile convolved with an exponential function which results in a slow decaying right tail of the profile.

After applying a matched filter with a Gaussian profile, we re-bin the data with different smoothing levels, from 0 to 11 (mentioned in section 4.1). Then we calculated SNR for all 12 smoothing levels and pick the maximum SNR A typical value of smoothing which results in a maximal SNR is between 4 and 7. When the maximum SNR appears at a smoothing level between 8 and 11, the $\mathrm{SNR}$ is not accurate, because the data are over-smoothed. For example, the 1023.56 sec pulse in RR0037_0011 data file has a SNR value of 19, but its smoothing level is 8. It was not detected by the conventional algorithm, and was considered to be noise.

To have a second opinion about a value of SNR, we involved a filter with rectangular profile. After applying this filter, we did not re-bin the data with different smoothing level, so that there is only one $\mathrm{SNR}$ calculated for each candidate. Besides the candidates with smoothing level over 7, the SNR calculated by both filters are identical. Taking the RR0037_0151 data file as an example, the average difference in $[\mathrm{SNR}$ between a matched filter based on a Gaussian profile and a rectangular profile is 1.3 .

\subsection{Candidate Pulses Detected in a Window Size of either $16 \mathrm{x}$ 16 or $32 \times 32$, but not in Both}

Apart from the pulses detected by the ETD algorithm and listed in Tables 4.7 through 4.16 there are 11 candidate pulses detected by the conventional algorithm, but not detected by the ETD algorithm. Also, there are 9 candidate pulses detected in the SFT space when the size of the 


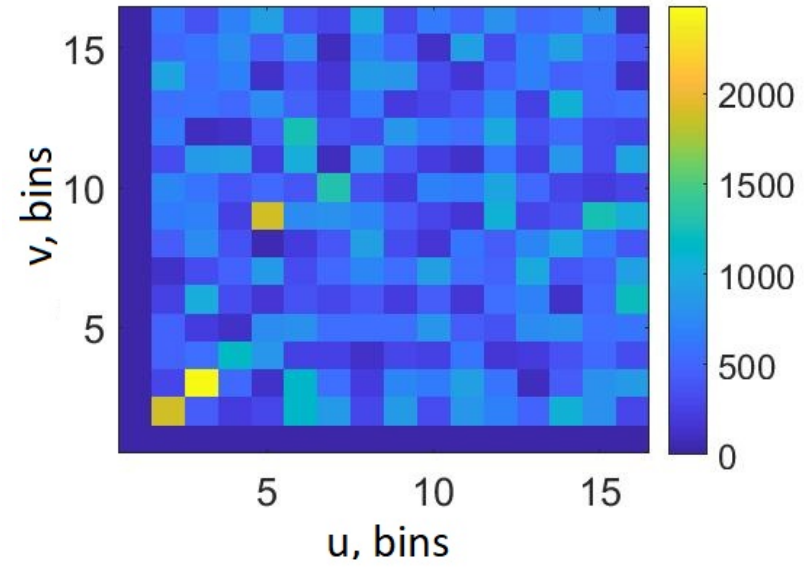

(a) window size $16 \times 16$

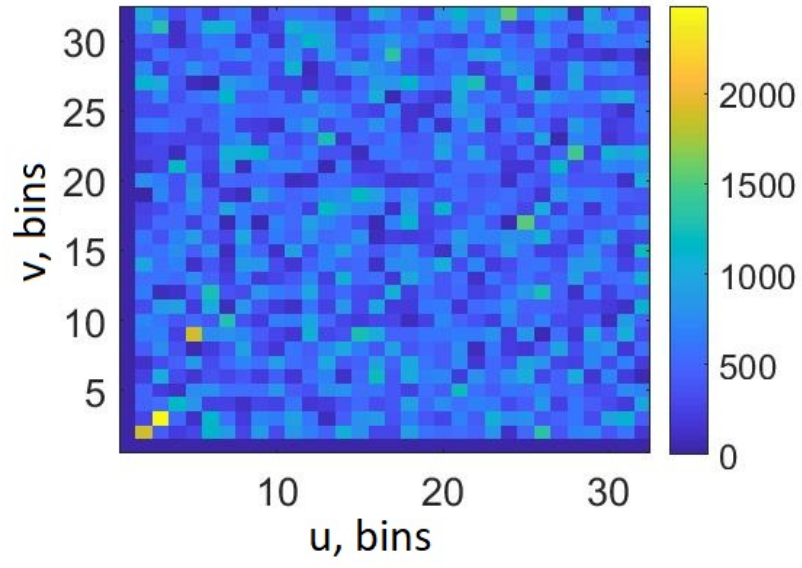

(b) window size $32 \times 32$

Figure 4.8: Two examples of a Fourier transformed spectrogram. Both figures show the SFT signature of a pulse of J1819-1548 arrived at 11005.3497 sec in RR0037_0151 data file. The left panel shows the SFT signature in only $16 \times 16$ window size, and the right panel shows the SFT signature in only $32 \times 32$ window size.

$(\mathrm{u}, \mathrm{v})$ window was set to $16 \times 16$, and 2 more candidate pulses are detected in the $\mathrm{SFT}$ space when the size of the $(\mathrm{u}, \mathrm{v})$ window was set to $32 \times 32$. The pulses are listed in Table 4.4 and Table 4.5 , respectively. The algorithm performing MF-SFT confirmed the 11 candidate pulses detected by the ETD algorithm. To illustrate one of the pulses detected by the ETD algorithm with the window size $16 \times 16$ but not with the window size $32 \times 32$, Figure 4.8 shows an example of the $\mathrm{SFT}$ signature of a pulse of J1819-1548. Note that most of the pulse signature is concentrated around the origin of the $\underline{\text { SFT }}$ space and thus the window of size $32 \times 32$ will include more noise pixels than the window size of $16 \times 16$. On the other hand in the case of a pulse with a narrow profile in the filterbank format, its reciprocal signature in the SFT space will stretch over a larger window and the window of size $16 \times 16$ will not account for the energy of all pixels in the pulse signature. In this case applying the window of size $32 \times 32$ leads to more reliable detection results.

\subsection{Broad Pulses}

The Fourier Transform of a rectangular pulse is a sinc function. Its first zero is observed at $N / W$ bin. The sinc function has a main lobe and side lobes. Most of energy is concentrated in its 


\begin{tabular}{|c|c|c|c|c|c|c|}
\hline File Name & TOA(s) & SNR & SNR2 & $\mathrm{CA}$ & ETD & MF-SFT \\
\hline RR0037_0151 & 1105.35 & 7.5 & 5.9 & $\mathrm{X}$ & - & $\mathrm{X}$ \\
\hline RR0037_0151 & 1326.97 & 6.2 & 7.0 & $X$ & - & $\mathrm{X}$ \\
\hline RR0037_0151 & 1391.02 & 6.3 & 5.0 & $X$ & - & $X$ \\
\hline RR0086_0181 & 1417.30 & 6.0 & 8.8 & $X$ & - & $X$ \\
\hline RR0086_0181 & 1489.31 & 8.5 & 7.0 & $X$ & - & $X$ \\
\hline RR0090_0121 & 602.24 & 5.2 & 5.4 & $X$ & - & $X$ \\
\hline RR0090_0121 & 680.84 & 6.4 & 5.8 & $\mathrm{X}$ & - & $\mathrm{X}$ \\
\hline RR0090_0121 & 686.24 & 9.1 & 13.5 & $\mathrm{X}$ & - & $\mathrm{X}$ \\
\hline RR0090_0181 & 1771.41 & 5.5 & 4.8 & $X$ & - & $X$ \\
\hline
\end{tabular}

Table 4.4: List of candidate pulses of the conventional algorithm detected by ETD only in the SFT $16 \times 16$ matrix. SNR2 is SNR calculated for the rectangular pulse.

\begin{tabular}{|c|c|c|c|c|c|c|}
\hline File Name & TOA(s) & SNR & SNR2 & CA & ETD & MF-SFT \\
\hline \hline RR0037_0151 & 1578.56 & 11.6 & 9.5 & $X$ & - & $X$ \\
\hline RR0090_0271 & 313.11 & 5.0 & 3.5 & $X$ & - & $X$ \\
\hline
\end{tabular}

Table 4.5: List of candidate pulses of the conventional algorithm detected by ETD only in the SFT $32 \times 32$ matrix. SNR2 is SNR calculated for rectangular pulse. 

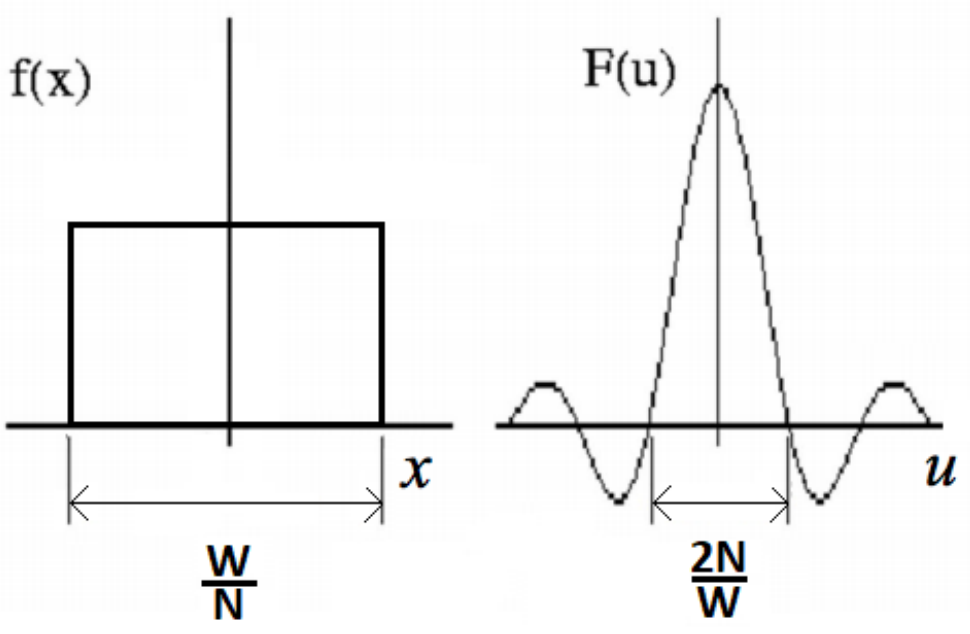

Figure 4.9: The left plot shows a rectangular pulse A.1. The right plot shows its Fourier Transform A.2.

main lobe (that is within $N / W$ bins). It is interesting to note that the larger width (W) is, the more concentrated energy is around the origin in the SFTdomain. Figure 4.9 illustrates this. Rectangular pulse A.1 is shown on the left panel and its Fourier Transform A.2 is shown on the right panel. As $\mathrm{W}$ grows, the pulse becomes broader and the value of $2 \mathrm{~N} / \mathrm{W}$ become smaller.

We also observed an example of extremely narrow pulses. Some are as narrow as a few pixels in width. Figure 4.11 presents two candidate pulses from the RRATs time series RR0070_0131 of J1423-5647. The pulse at the top has SNR of 21.5 and estimated width of $12.8 \mathrm{~ms}$. It is detected by both the conventional algorithm and by the ETD algorithm. The pulse at the bottom has SNR of 7.17 and estimated width of $12.8 \mathrm{~ms}$. It is detected by only the ETD algorithm in the window of size $16 \times 16$. It is not detected by the conventional algorithm with the threshold set to $\mathrm{SNR}=5$ (this candidate was not detected as a pulse). Note that the two pulses are broad in time series and very concentrated in the lower left corner of the SFT domain. They have the same DM value (based on the slope of dispersed pulses) and a very similar signature in the SFT domain. In addition, both pulses are detected in smaller window size of $8 \times 8$.

For a deeper analysis of the detection performance of the ETD algorithm in Table 4.6 we displayed the SNR values of all pulses in RR0037_0151 detected by the conventional algorithm versus the estimated width of each pulse. Figure 4.12 demonstrates this dependence. We colorcoded all pulses based on whether or not the ETD algorithm also detects them. A pulse represented 


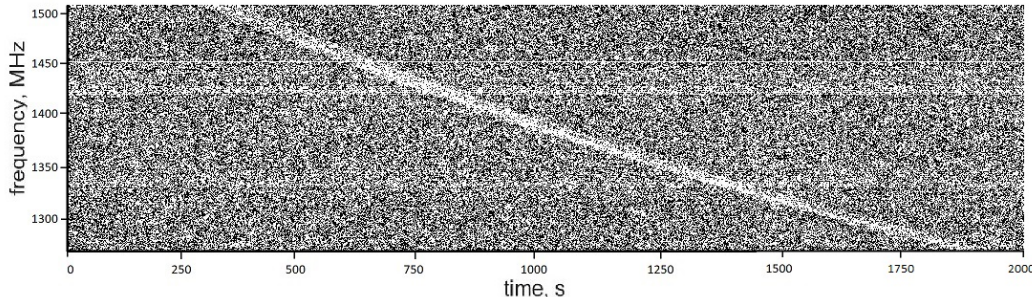

(a)

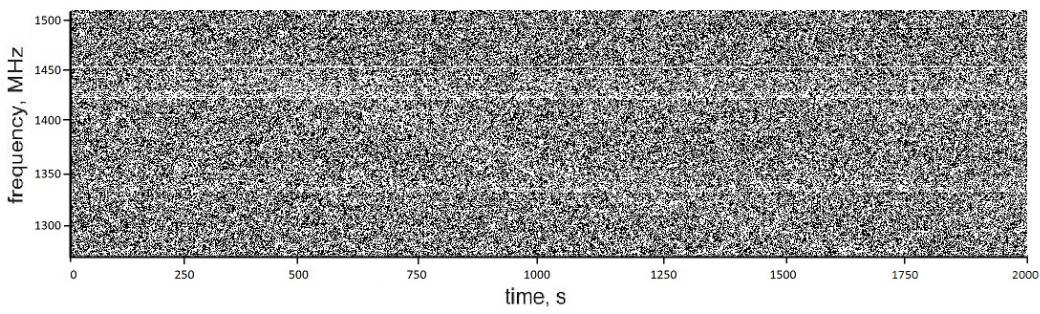

(c)

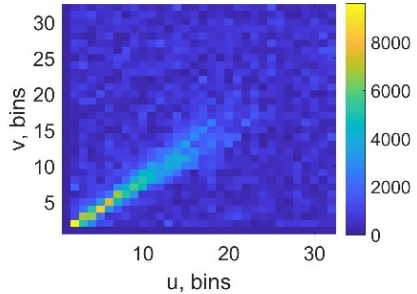

(b)

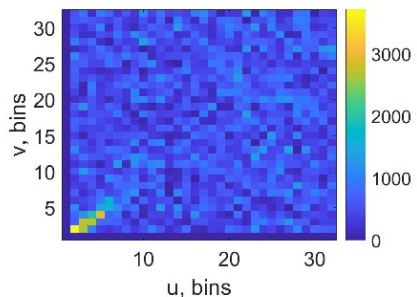

(d)

Figure 4.10: Panel (a) shows a spectrogram of the pulse timed at 440.1873 sec. in RR0037_0151. The pulse is detected by both the conventional algorithm and by the ETD algorithm. Panel (b) shows the signature of this pulse in the SFT domain. Panel (c) shows the spectrogram of the pulse at $423.0801 \mathrm{sec}$. in RR0037_0151. The pulse is detected by both the conventional algorithm and by the ETD algorithm. Panel (d) shows the signature of the pulse in (c) in the SFT domain.

by a red square is not detected by the ETD algorithm. A pulse marked with a blue diamond represents a detection. Note that the ETD algorithm fails to detect almost all pulses of width 0.8 ms. Only one pulse, with the largest $\mathrm{SNR}$ among all $0.8 \mathrm{~ms}$ width pulses, is detected in a window of size $32 \times 32$. As the width of pulses increases, the ETD algorithm is able to detect more and more pulses. There are 2 pulses of width $25.6 \mathrm{~ms}$, but only one of them is detected in both types of window. The other pulse with a lower $\mathrm{SNR}$ value is detected only by the window of size $16 \times 16$. 


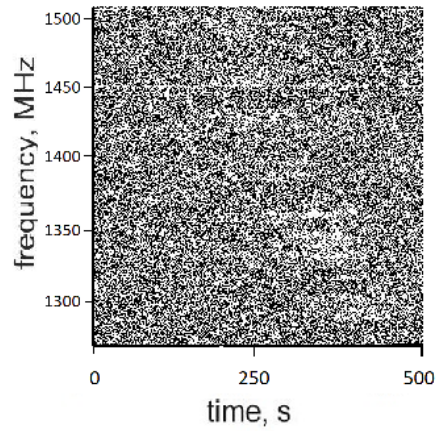

(a)

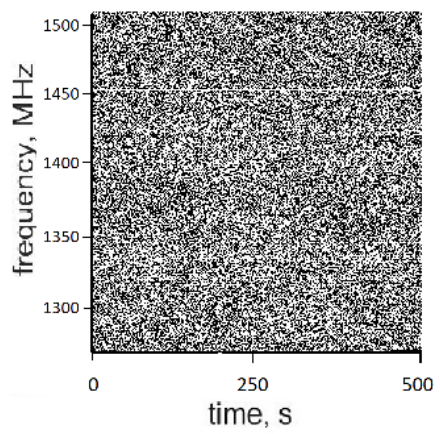

(c)

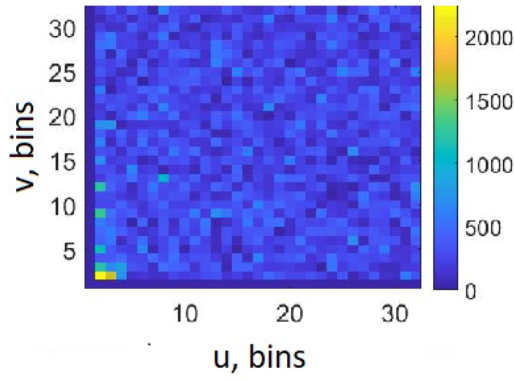

(b)

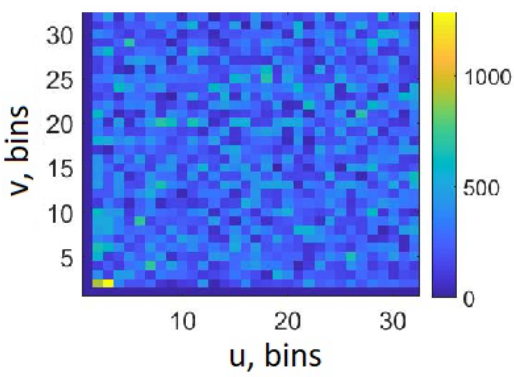

(d)

Figure 4.11: Panel (a) shows the spectrogram of the pulse timed at 331.3901 s in RR0070_0131. The pulse is detected by both the conventional algorithm and by the ETD algorithm. Panel (b) displays the signature of this pulse in the SFT domain. Panel (c) shows the spectrogram of the pulse at 198.5001 s in RR0070_0131. The pulse is detected by only the ETD algorithm in 16×16 window size, but not detected by the conventional algorithm. Panel (d) displays the signature of this pulse in the SFT domain. 


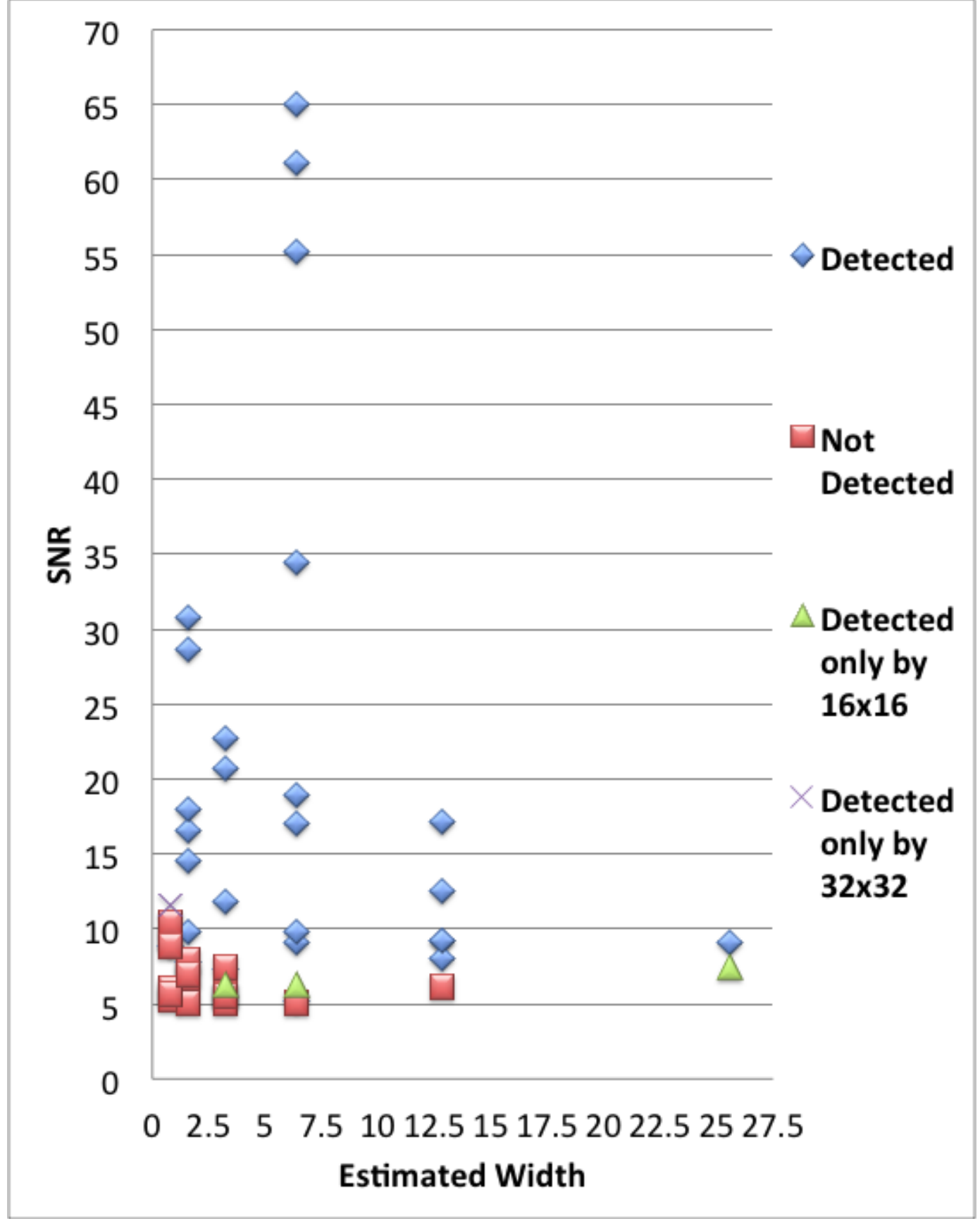

Figure 4.12: A plot of SNR values of pulses detected by the conventional algorithm versus the estimated width of each pulse. Blue diamonds indicate pulses detected by the ETD algorithm. Red squares indicate pulses missed by the ETD algorithm. Green triangles are pulses detected by the ETD algorithm but in a window of size $16 \times 16$. Purple crosses mark pulses detected by the ETD algorithm in a window of size $32 \times 32$. 


\begin{tabular}{|c|c|c|c|c|c|c|c|}
\hline Width & $\begin{array}{c}\text { Number of } \\
\text { Candidates }\end{array}$ & Detections & $\begin{array}{c}\text { Not } \\
\text { Detected }\end{array}$ & $\begin{array}{c}\text { Detected only } \\
\text { by } 16 x 16\end{array}$ & $\begin{array}{c}\text { Detected only } \\
\text { by } 32 \times 32\end{array}$ & $\begin{array}{c}\text { Detection } \\
\text { Rate }\end{array}$ & $\begin{array}{c}\text { Detection Rate including } \\
16 \times 16 \text { or 32x32 only }\end{array}$ \\
\hline \hline 25.6 & 2 & 1 & 1 & 1 & 0 & $50.0 \%$ & $100.0 \%$ \\
\hline 12.8 & 6 & 5 & 1 & 0 & 0 & $83.3 \%$ & $83.3 \%$ \\
\hline 6.4 & 11 & 9 & 2 & 1 & 0 & $81.8 \%$ & $90.9 \%$ \\
\hline 3.21 & 10 & 4 & 6 & 1 & 0 & $40.0 \%$ & $50.0 \%$ \\
\hline 1.6 & 11 & 7 & 4 & 0 & 0 & $63.6 \%$ & $63.6 \%$ \\
\hline 0.8 & 8 & 1 & 7 & 0 & 1 & $12.5 \%$ & $25.0 \%$ \\
\hline Sum & 48 & 27 & 21 & 3 & 1 & $56.3 \%$ & $64.6 \%$ \\
\hline
\end{tabular}

Table 4.6: The table presents a summary of the detection performance by the three detection algorithms described in the previous chapter. The table lists estimated width of pulse, number of candidate pulses, number of pulses detected by ETD, number of pulses not detected by ETD (neither in $16 \times 16$ nor $32 \times 32$ window size), number of pulses detected only in $16 \times 16$ window size, number of pulses detected only in $32 \times 32$ window size, detection rate of ETD, and detection rate if counting the pulses detected only in $16 \times 16$ or $32 \times 32$ window size. Each entry of the last row in the table is a sum of values along each column. The last two entries of the last row present the average of columns 7 and 8 .

\begin{tabular}{|c|c|c|c|c|c|}
\hline TOA (s) & SNR & SNR2 & CA & ETD & MF-SFT \\
\hline \hline 911.34 & 3.9 & 2.7 & - & $\mathrm{X}$ & $\mathrm{X}$ \\
\hline 994.51 & 5.2 & 3.1 & $\mathrm{X}$ & - & - \\
\hline 1023.56 & 19.0 & 2.8 & - & $\mathrm{X}$ & - \\
\hline 1067.28 & 5.4 & 4.3 & $\mathrm{X}$ & - & - \\
\hline 1110.76 & 4.1 & 2.4 & - & $\mathrm{X}$ & - \\
\hline 1135.94 & 3.1 & 2.0 & - & $\mathrm{X}$ & - \\
\hline 1394.66 & 4.9 & 2.7 & - & $\mathrm{X}$ & - \\
\hline 1476.36 & 5.2 & 4.1 & $\mathrm{X}$ & - & - \\
\hline 1918.48 & 3.7 & 2.0 & - & $\mathrm{X}$ & $\mathrm{X}$ \\
\hline 2145.54 & 4.1 & 3.4 & - & $\mathrm{X}$ & $\mathrm{X}$ \\
\hline 2361.84 & 5.3 & 2.8 & $\mathrm{X}$ & - & $\mathrm{X}$ \\
\hline 2628.78 & 5.4 & 6.1 & $\mathrm{X}$ & - & $\mathrm{X}$ \\
\hline 2792.50 & 5.2 & 4.5 & $\mathrm{X}$ & - & $\mathrm{X}$ \\
\hline 3176.56 & 4.0 & 2.5 & - & $\mathrm{X}$ & $\mathrm{X}$ \\
\hline
\end{tabular}

Table 4.7: A summary of the detection performance by the three detection algorithms introduced in the previous chapter. The algorithms were applied to J1754-3011 data file of RR0037_0011. SNR2 is an SNR calculated using a rectangular pulse profile. 


\begin{tabular}{|c|c|c|c|c|c|}
\hline TOA (s) & SNR & SNR2 & $\mathrm{CA}$ & ETD & MF-SFT \\
\hline 19.75 & 3.4 & 3.3 & - & $\mathrm{X}$ & - \\
\hline 34.60 & 3.8 & 3.1 & - & $X$ & $X$ \\
\hline 74.85 & 2.9 & 3.1 & - & $\mathrm{X}$ & $\mathrm{X}$ \\
\hline 87.90 & 3.2 & 2.9 & - & $\mathrm{X}$ & $\mathrm{X}$ \\
\hline 211.05 & 3.2 & 3.3 & - & $\mathrm{X}$ & $X$ \\
\hline 277.30 & 3.6 & 2.6 & - & $\mathrm{X}$ & $\mathrm{X}$ \\
\hline 304.40 & 3.0 & 2.8 & - & $\mathrm{X}$ & $X$ \\
\hline 484.40 & 3.1 & 2.8 & - & $\mathrm{X}$ & $X$ \\
\hline 656.95 & 4.3 & 3.1 & - & $\mathrm{X}$ & $X$ \\
\hline 694.35 & 3.2 & 2.6 & - & $\mathrm{X}$ & $X$ \\
\hline 736.25 & 3.2 & 2.5 & - & $X$ & $X$ \\
\hline 736.30 & 4.7 & 2.6 & - & $\mathrm{X}$ & $\mathrm{X}$ \\
\hline 813.70 & 4.0 & 2.4 & - & $\mathrm{X}$ & $X$ \\
\hline 842.55 & 3.9 & 2.8 & - & $X$ & $X$ \\
\hline 855.65 & 3.0 & 3.4 & - & $\mathrm{X}$ & $X$ \\
\hline 874.55 & 3.3 & 4.1 & - & $X$ & $X$ \\
\hline 959.40 & 3.2 & 2.7 & - & $X$ & $X$ \\
\hline 1045.95 & 4.2 & 3.4 & - & $\mathrm{X}$ & $\mathrm{X}$ \\
\hline 1073.10 & 3.3 & 3.7 & - & $X$ & $\mathrm{X}$ \\
\hline 1124.40 & 3.1 & 3.5 & - & $X$ & $X$ \\
\hline 1149.55 & 3.7 & 2.6 & - & $\mathrm{X}$ & $\mathrm{X}$ \\
\hline 1214.20 & 3.5 & 2.8 & - & $X$ & $X$ \\
\hline 1352.10 & 3.4 & 3.0 & - & $\mathrm{X}$ & $\mathrm{X}$ \\
\hline 1354.90 & 3.3 & 3.2 & - & $\mathrm{X}$ & $\mathrm{X}$ \\
\hline 1365.15 & 2.9 & 3.1 & - & $\mathrm{X}$ & $\mathrm{X}$ \\
\hline 1367.00 & 3.5 & 3.3 & - & $\mathrm{X}$ & $X$ \\
\hline 1372.69 & 5.2 & 5.3 & $\mathrm{X}$ & - & $X$ \\
\hline 1500.65 & 3.1 & 3.2 & - & $\mathrm{X}$ & $X$ \\
\hline 1573.30 & 3.9 & 2.7 & - & $\mathrm{X}$ & $X$ \\
\hline 1665.55 & 3.1 & 5.1 & - & $\mathrm{X}$ & $X$ \\
\hline 1722.40 & 3.0 & 2.9 & - & $X$ & $\mathrm{X}$ \\
\hline
\end{tabular}

Table 4.8: A summary of the detection performance by the three detection algorithms introduced in the previous chapter. The algorithms were applied to J1839-0136 data file of RR0037_0031. SNR2 is an SNR calculated using a rectangular pulse profile. 


\begin{tabular}{|c|c|c|c|c|c|}
\hline TOA (s) & SNR & SNR2 & $\mathrm{CA}$ & ETD & MF-SFT \\
\hline 70.74 & 10.4 & 2.2 & - & $\mathrm{X}$ & - \\
\hline 147.24 & 5.1 & 2.9 & $X$ & - & - \\
\hline 152.67 & 3.4 & 2.0 & - & $X$ & - \\
\hline 171.18 & 2.6 & 3.2 & - & $X$ & $X$ \\
\hline 195.00 & 3.8 & 2.9 & - & $\mathrm{X}$ & $\mathrm{X}$ \\
\hline 197.64 & 3.6 & 3.1 & - & $X$ & $X$ \\
\hline 297.81 & 3.9 & 3.0 & - & $X$ & $X$ \\
\hline 303.06 & 3.4 & 3.0 & - & $X$ & - \\
\hline 305.76 & 2.8 & 2.6 & - & $\mathrm{X}$ & $\mathrm{X}$ \\
\hline 421.95 & 2.7 & 3.0 & - & $\mathrm{X}$ & $\mathrm{X}$ \\
\hline 432.60 & 3.4 & 2.7 & - & $\mathrm{X}$ & $\mathrm{X}$ \\
\hline 517.00 & 19.6 & 22.8 & $\mathrm{X}$ & $\mathrm{X}$ & $\mathrm{X}$ \\
\hline 519.63 & 9.7 & 3.3 & - & $X$ & $X$ \\
\hline 524.88 & 3.0 & 3.2 & - & $X$ & - \\
\hline 532.77 & 3.8 & 2.7 & - & $\mathrm{X}$ & - \\
\hline 588.34 & 9.8 & 11.7 & $X$ & $X$ & $X$ \\
\hline 612.12 & 6.9 & 3.2 & - & $X$ & - \\
\hline 635.88 & 3.2 & 2.9 & - & $\mathrm{X}$ & - \\
\hline 649.11 & 7.5 & 6.3 & $X$ & $\mathrm{X}$ & $X$ \\
\hline 670.32 & 3.6 & 2.7 & - & $X$ & $X$ \\
\hline 770.61 & 4.5 & 4.8 & - & $\mathrm{X}$ & - \\
\hline 773.31 & 11.8 & 12.1 & $X$ & $\mathrm{X}$ & $X$ \\
\hline 828.81 & 15.9 & 12.9 & $X$ & $X$ & $X$ \\
\hline 831.43 & 6.0 & 7.0 & $X$ & - & $\mathrm{X}$ \\
\hline 836.72 & 5.3 & 7.2 & $X$ & - & - \\
\hline 839.37 & 3.6 & 2.9 & - & $\mathrm{X}$ & - \\
\hline 847.17 & 4.2 & 2.4 & - & $X$ & - \\
\hline 857.88 & 4.8 & 4.2 & - & $X$ & - \\
\hline 971.28 & 4.2 & 3.4 & - & $X$ & - \\
\hline 1013.75 & 13.2 & 11.6 & $X$ & $X$ & $X$ \\
\hline 1024.32 & 3.8 & 2.8 & - & $X$ & $\mathrm{X}$ \\
\hline 1048.02 & 2.8 & 2.4 & - & $X$ & - \\
\hline 1108.71 & 2.7 & 2.4 & - & $\mathrm{X}$ & $\mathrm{X}$ \\
\hline 1177.38 & inf & 2.3 & - & $\mathrm{X}$ & - \\
\hline 1267.08 & 3.0 & 3.2 & - & $X$ & - \\
\hline 1288.32 & 4.1 & 3.2 & - & $X$ & - \\
\hline 1341.18 & 3.7 & 3.1 & - & $X$ & - \\
\hline 1372.83 & 3.1 & 2.7 & - & $X$ & $\mathrm{X}$ \\
\hline 1380.72 & 3.6 & 2.8 & - & $\mathrm{X}$ & - \\
\hline 1396.53 & 31.9 & 2.4 & - & $\mathrm{X}$ & - \\
\hline 1431.20 & 9.9 & 7.3 & $X$ & $X$ & $X$ \\
\hline 1552.56 & 2.4 & 2.5 & - & $X$ & - \\
\hline 1573.74 & 3.4 & 2.9 & - & $X$ & $X$ \\
\hline 1642.32 & 3.0 & 3.7 & - & $X$ & $\mathrm{X}$ \\
\hline 1655.81 & 6.8 & 6.3 & $X$ & - & $\mathrm{X}$ \\
\hline 1661.11 & 5.6 & 6.5 & $\mathrm{X}$ & - & $X$ \\
\hline 1758.86 & 5.4 & 4.5 & $X$ & - & $X$ \\
\hline
\end{tabular}

Table 4.9: A summary of the detection performance by the three detection algorithms introduced in the previous chapter. The algorithms were applied to J1317-5759 data file of RR0037_0071. SNR2 is an SNR calculated using a rectangular pulse profile. 


\begin{tabular}{|c|c|c|c|c|c|}
\hline TOA (s) & SNR & SNR2 & $\mathrm{CA}$ & ETD & MF-SFT \\
\hline 9.53 & 6.0 & 5.1 & $\mathrm{X}$ & - & $\mathrm{X}$ \\
\hline 116.14 & 9.1 & 7.3 & $\mathrm{X}$ & X & $\mathrm{X}$ \\
\hline 256.81 & $\mid 34.4$ & 30.0 & $\mathrm{X}$ & $X$ & $\mathrm{X}$ \\
\hline 291.00 & 3.8 & 5.3 & - & $\mathrm{X}$ & $\mathrm{X}$ \\
\hline 312.29 & 9.3 & 7.5 & $\mathrm{X}$ & $\mathrm{X}$ & $\mathrm{X}$ \\
\hline 388.77 & 5.1 & 4.2 & $X$ & - & $\mathrm{X}$ \\
\hline 423.08 & 17.2 & 11.8 & $\mathrm{X}$ & $\mathrm{X}$ & $\mathrm{X}$ \\
\hline 427.40 & 20.7 & 18.7 & $\mathrm{X}$ & $\mathrm{X}$ & $\mathrm{X}$ \\
\hline 440.19 & 65.1 & 54.4 & $\mathrm{X}$ & $\mathrm{X}$ & $\mathrm{X}$ \\
\hline 444.47 & 11.8 & 13.8 & $\mathrm{X}$ & $\mathrm{X}$ & $\mathrm{X}$ \\
\hline 448.74 & 7.4 & 7.4 & $\mathrm{X}$ & - & $\mathrm{X}$ \\
\hline 457.30 & 6.2 & 6.2 & $\mathrm{X}$ & - & $\mathrm{X}$ \\
\hline 461.55 & 18.9 & 19.5 & $\mathrm{X}$ & $\mathrm{X}$ & $\mathrm{X}$ \\
\hline 555.35 & 9.1 & 6.9 & $\mathrm{X}$ & $\mathrm{X}$ & $\mathrm{X}$ \\
\hline 597.94 & 9.5 & 7.2 & $\mathrm{X}$ & - & $\mathrm{X}$ \\
\hline 781.32 & 7.4 & 6.4 & $\mathrm{X}$ & $\mathrm{X}$ & $\mathrm{X}$ \\
\hline 807.09 & 5.3 & 3.6 & $\mathrm{X}$ & - & $\mathrm{X}$ \\
\hline 883.59 & 7.8 & 7.8 & $\mathrm{X}$ & $\mathrm{X}$ & $\mathrm{X}$ \\
\hline 900.65 & 55.2 & 51.6 & $\mathrm{X}$ & $\mathrm{X}$ & $\mathrm{X}$ \\
\hline 934.82 & 9.8 & 9.8 & $\mathrm{X}$ & $\mathrm{X}$ & $\mathrm{X}$ \\
\hline 1016.14 & 5.1 & 4.0 & $\mathrm{X}$ & - & - \\
\hline 1079.72 & \begin{tabular}{|l|}
30.7 \\
\end{tabular} & 36.4 & $\mathrm{X}$ & $\mathrm{X}$ & $\mathrm{X}$ \\
\hline 1083.99 & 28.6 & 22.9 & $\mathrm{X}$ & $\mathrm{X}$ & $\mathrm{X}$ \\
\hline 1096.78 & 10.5 & 7.9 & $\mathrm{X}$ & - & $\mathrm{X}$ \\
\hline 1101.08 & 6.7 & 6.2 & $\mathrm{X}$ & - & $\mathrm{X}$ \\
\hline 1105.35 & 7.5 & 5.9 & $X$ & - & $\mathrm{X}$ \\
\hline 1186.30 & 5.6 & 7.0 & $\mathrm{X}$ & - & $\mathrm{X}$ \\
\hline 1216.22 & 5.7 & 6.3 & $X$ & - & $\mathrm{X}$ \\
\hline 1220.48 & 5.1 & 5.4 & $\mathrm{X}$ & - & $\mathrm{X}$ \\
\hline 1237.42 & 9.8 & 9.0 & $\mathrm{X}$ & $\mathrm{X}$ & $\mathrm{X}$ \\
\hline 1241.70 & 8.0 & 8.5 & $\mathrm{X}$ & - & $\mathrm{X}$ \\
\hline 1322.70 & 16.5 & 12.5 & $\mathrm{X}$ & $\mathrm{X}$ & $\mathrm{X}$ \\
\hline 1326.97 & 6.2 & 7.0 & $X$ & - & $\mathrm{X}$ \\
\hline 1331.27 & 61.2 & 42.0 & $\mathrm{X}$ & $\mathrm{X}$ & $\mathrm{X}$ \\
\hline 1348.35 & \begin{tabular}{|l|}
17.0 \\
\end{tabular} & 18.6 & $\mathrm{X}$ & $\mathrm{X}$ & $\mathrm{X}$ \\
\hline 1352.66 & 5.6 & 6.4 & $\mathrm{X}$ & - & $\mathrm{X}$ \\
\hline 1356.85 & 14.6 & 7.9 & $\mathrm{X}$ & $x$ & $\mathrm{X}$ \\
\hline 1365.38 & 5.5 & 7.8 & $\mathrm{X}$ & - & $\mathrm{X}$ \\
\hline 1369.70 & 22.7 & 21.9 & $\mathrm{X}$ & $\mathrm{X}$ & $\mathrm{X}$ \\
\hline 1386.79 & 5.2 & 6.8 & $\mathrm{X}$ & $\mathrm{X}$ & $\mathrm{X}$ \\
\hline 1391.02 & 6.3 & 5.0 & $\mathrm{X}$ & - & $\mathrm{X}$ \\
\hline 1429.27 & 7.0 & 7.0 & $\mathrm{X}$ & - & $\mathrm{X}$ \\
\hline 1459.32 & 3.5 & 3.4 & - & $\mathrm{X}$ & $\mathrm{X}$ \\
\hline 1463.49 & 8.8 & 7.7 & $X$ & - & $\mathrm{X}$ \\
\hline 1467.71 & 18.0 & 24.4 & $\mathrm{X}$ & $\mathrm{X}$ & $\mathrm{X}$ \\
\hline 1480.56 & 8.0 & 8.2 & $\mathrm{X}$ & $\mathrm{X}$ & $\mathrm{X}$ \\
\hline 1578.56 & 11.6 & 9.5 & $\mathrm{X}$ & - & $\mathrm{X}$ \\
\hline 1612.71 & 12.5 & 11.8 & $\mathrm{X}$ & $\mathrm{X}$ & $\mathrm{X}$ \\
\hline 1625.52 & 9.2 & 8.6 & $\mathrm{X}$ & $\mathrm{X}$ & $\mathrm{X}$ \\
\hline 1761.85 & 8.8 & 4.4 & $\mathrm{X}$ & $\mathrm{X}$ & $\mathrm{X}$ \\
\hline 1791.84 & 8.4 & 8.1 & - & $\mathrm{X}$ & $\mathrm{X}$ \\
\hline
\end{tabular}

Table 4.10: A summary of the detection performance by the three detection algorithms introduced in the previous chapter. The algorithms were applied to J1819-1458 data file of RR0037_0151. SNR2 is an SNR calculated using a rectangular pulse profile. 


\begin{tabular}{|c|c|c|c|c|c|}
\hline TOA (s) & SNR & SNR2 & CA & ETD & MF-SFT \\
\hline \hline 331.40 & 21.5 & 7.2 & $\mathrm{X}$ & $\mathrm{X}$ & $\mathrm{X}$ \\
\hline 1602.84 & 3.6 & 3.4 & - & $\mathrm{X}$ & $\mathrm{X}$ \\
\hline
\end{tabular}

Table 4.11: A summary of the detection performance by the three detection algorithms introduced in the previous chapter. The algorithms were applied to J1423-5647 data file of RR0070_0131. SNR2 is an SNR calculated using a rectangular pulse profile.

\begin{tabular}{|c|c|c|c|c|c|}
\hline TOA (s) & SNR & SNR2 & CA & ETD & MF-SFT \\
\hline \hline 446.34 & 7.3 & 2.6 & - & $\mathrm{X}$ & $\mathrm{X}$ \\
\hline 887.58 & 3.6 & 2.7 & - & $\mathrm{X}$ & $\mathrm{X}$ \\
\hline 1260.36 & 5.3 & 3.0 & $\mathrm{X}$ & - & - \\
\hline 1408.06 & 16.1 & 17.1 & $\mathrm{X}$ & $\mathrm{X}$ & $\mathrm{X}$ \\
\hline 1408.99 & 6.7 & 7.3 & $\mathrm{X}$ & - & $\mathrm{X}$ \\
\hline 1417.30 & 6.0 & 8.8 & $\mathrm{X}$ & - & $\mathrm{X}$ \\
\hline 1420.99 & 6.8 & 7.3 & $\mathrm{X}$ & $\mathrm{X}$ & $\mathrm{X}$ \\
\hline 1489.31 & 8.5 & 7.0 & $\mathrm{X}$ & - & $\mathrm{X}$ \\
\hline 1642.54 & 5.1 & 5.4 & $\mathrm{X}$ & - & $\mathrm{X}$ \\
\hline 1757.94 & 4.1 & 3.2 & - & $\mathrm{X}$ & $\mathrm{X}$ \\
\hline
\end{tabular}

Table 4.12: The performance of the three algorithms for J1913+1330 data file RR0086_0181's candidate pulses. SNR2 is SNR calculated for rectangle pulse profile. 


\begin{tabular}{|c|c|c|c|c|c|}
\hline TOA (s) & SNR & SNR2 & $\mathrm{CA}$ & ETD & MF-SFT \\
\hline 26.19 & 2.7 & 3.0 & - & $\mathrm{X}$ & - \\
\hline 158.04 & 2.7 & 3.3 & - & $\mathrm{X}$ & $\mathrm{X}$ \\
\hline 185.79 & inf & 3.0 & - & $\mathrm{X}$ & $\mathrm{X}$ \\
\hline 200.49 & 3.6 & 2.8 & - & $\mathrm{X}$ & $\mathrm{X}$ \\
\hline 251.34 & 3.3 & 3.2 & - & X & X \\
\hline 290.79 & 2.9 & 2.6 & - & $\mathrm{X}$ & X \\
\hline 335.52 & 3.0 & 3.2 & - & $\mathrm{X}$ & - \\
\hline 341.61 & 2.7 & 3.5 & - & X & - \\
\hline 395.58 & 5.5 & 3.5 & $X$ & - & $X$ \\
\hline 400.14 & 4.0 & 3.8 & - & $X$ & - \\
\hline 422.58 & 2.7 & 3.1 & - & X & $\mathrm{X}$ \\
\hline 495.81 & 3.0 & 3.0 & - & X & $X$ \\
\hline 561.24 & 3.3 & 3.6 & - & $\mathrm{X}$ & $\mathrm{X}$ \\
\hline 567.45 & 3.1 & 2.8 & - & $\mathrm{X}$ & $\mathrm{X}$ \\
\hline 595.32 & 4.1 & 2.5 & - & $\mathrm{X}$ & $\mathrm{X}$ \\
\hline 600.69 & 6.1 & 5.9 & $X$ & - & $\mathrm{X}$ \\
\hline 602.24 & 5.2 & 5.4 & $X$ & - & X \\
\hline 608.41 & 6.7 & 5.6 & X & - & $\mathrm{X}$ \\
\hline 609.18 & 7.5 & 8.6 & $X$ & $\mathrm{X}$ & X \\
\hline 609.95 & 5.7 & 7.4 & $X$ & - & $\mathrm{X}$ \\
\hline 635.31 & 4.0 & 3.7 & - & $\mathrm{X}$ & $\mathrm{X}$ \\
\hline 636.92 & 7.3 & 8.9 & $X$ & - & $\mathrm{X}$ \\
\hline 644.63 & 10.4 & 10.5 & $X$ & X & $\mathrm{X}$ \\
\hline 645.40 & 10.5 & 12.9 & X & X & $\mathrm{X}$ \\
\hline 661.59 & 3.4 & 2.9 & - & X & $X$ \\
\hline 670.82 & 5.5 & 5.1 & $X$ & - & $\mathrm{X}$ \\
\hline 680.84 & 6.4 & 5.8 & $X$ & - & $X$ \\
\hline 686.24 & 9.1 & 13.5 & $X$ & - & $\mathrm{X}$ \\
\hline 693.95 & 6.4 & 7.2 & $X$ & - & - \\
\hline 694.72 & 5.7 & 6.2 & $\mathrm{X}$ & $\mathrm{X}$ & $X$ \\
\hline 697.03 & 8.2 & 8.1 & X & - & X \\
\hline 697.80 & 5.6 & 6.2 & X & - & - \\
\hline 707.76 & 3.0 & 3.0 & - & X & - \\
\hline 1008.51 & 9.6 & 3.0 & - & $\mathrm{X}$ & $\mathrm{X}$ \\
\hline 1169.67 & 2.9 & 3.1 & - & $\mathrm{X}$ & $\mathrm{X}$ \\
\hline
\end{tabular}

Table 4.13: The performance of the three algorithms for J1826-1419 data file RR0090_0121's candidate pulses. SNR2 is SNR calculated for rectangle pulse profile. 


\begin{tabular}{|c|c|c|c|c|c|}
\hline TOA (s) & SNR & SNR2 & CA & ETD & MF-SFT \\
\hline \hline 1771.41 & 5.5 & 4.8 & $\mathrm{X}$ & - & $\mathrm{X}$ \\
\hline 2038.21 & 14.8 & 9.8 & $\mathrm{X}$ & $\mathrm{X}$ & $\mathrm{X}$ \\
\hline
\end{tabular}

Table 4.14: The performance of the three algorithms for J1444-6026 data file RR0090_0181's candidate pulses. SNR2 is SNR calculated for rectangle pulse profile.

\begin{tabular}{|c|c|c|c|c|c|}
\hline TOA (s) & SNR & SNR2 & CA & ETD & MF-SFT \\
\hline \hline 588.68 & 2.9 & 3.0 & - & $\mathrm{X}$ & $\mathrm{X}$ \\
\hline 893.08 & 5.1 & 3.5 & $\mathrm{X}$ & - & - \\
\hline 1287.10 & 6.0 & 5.3 & $\mathrm{X}$ & - & $\mathrm{X}$ \\
\hline 1425.74 & 5.7 & 3.4 & $\mathrm{X}$ & - & - \\
\hline 3212.14 & 5.3 & 3.9 & $\mathrm{X}$ & - & $\mathrm{X}$ \\
\hline 3216.63 & 5.0 & 4.9 & $\mathrm{X}$ & - & $\mathrm{X}$ \\
\hline 3516.56 & 5.8 & 4.0 & $\mathrm{X}$ & - & $\mathrm{X}$ \\
\hline 3574.64 & 5.0 & 4.3 & $\mathrm{X}$ & - & $\mathrm{X}$ \\
\hline
\end{tabular}

Table 4.15: The performance of the three algorithms for J1846-0257 data file RR0090_0211's candidate pulses. SNR2 is SNR calculated for rectangle pulse profile.

\begin{tabular}{|c|c|c|c|c|c|}
\hline TOA (s) & SNR & SNR2 & CA & ETD & MF-SFT \\
\hline \hline 81.92 & 4.3 & 2.9 & - & $\mathrm{X}$ & $\mathrm{X}$ \\
\hline 313.11 & 5.0 & 3.5 & $\mathrm{X}$ & - & $\mathrm{X}$ \\
\hline 369.28 & 4.2 & 2.4 & - & $\mathrm{X}$ & $\mathrm{X}$ \\
\hline 376.36 & 4.8 & 2.5 & - & $\mathrm{X}$ & $\mathrm{X}$ \\
\hline 460.16 & 11.8 & 1.8 & - & $\mathrm{X}$ & - \\
\hline 574.64 & 2.8 & 2.8 & - & $\mathrm{X}$ & $\mathrm{X}$ \\
\hline 643.75 & 6.4 & 4.8 & $\mathrm{X}$ & - & $\mathrm{X}$ \\
\hline 994.68 & 3.5 & 3.3 & - & $\mathrm{X}$ & $\mathrm{X}$ \\
\hline 1025.60 & 5.7 & 3.5 & - & $\mathrm{X}$ & $\mathrm{X}$ \\
\hline 1049.27 & 6.0 & 3.7 & $\mathrm{X}$ & - & $\mathrm{X}$ \\
\hline 1094.52 & 6.0 & 3.7 & - & $\mathrm{X}$ & $\mathrm{X}$ \\
\hline 1232.84 & 16.2 & 2.8 & - & $\mathrm{X}$ & - \\
\hline 1392.64 & 4.0 & 2.4 & - & $\mathrm{X}$ & $\mathrm{X}$ \\
\hline 1612.72 & 11.8 & 2.9 & - & $\mathrm{X}$ & $\mathrm{X}$ \\
\hline 1650.88 & 3.2 & 2.7 & - & $\mathrm{X}$ & $\mathrm{X}$ \\
\hline
\end{tabular}

Table 4.16: The performance of the three algorithms for J1739-2513 data file RR0090_0271's candidate pulses. SNR2 is SNR calculated for rectangle pulse profile. 


\section{Chapter 5}

\section{Summary and Future Work}

In this final chapter, we present a summary of our results and discuss future directions for our work.

\subsection{Brief Summary}

In this thesis we proposed a new algorithm, which we called Energy in Transformed Data (ETD), for the detection of isolated astrophysical pulses. Unlike the conventional algorithm, the ETD algorithm detects pulses in a space reciprocal to the space of filterbank data. The ETD algorithm is implemented in three steps: (1) it applies the SFT to filterbank data, (2) it evaluates the signal energy in the transformed space, and (3) it compares the value of the total energy to a threshold. If the energy in transformed data exceeds the threshold, we claim that we detect a potential pulse. To analyze the detection capabilities of the ETD algorithm, we applied the algorithm to ten different RRAT time series. The performance of the algorithm was compared to the performance of two other algorithms one of which is the conventional algorithm and the other one is an algorithm that performs matched filtering in the SFT domain.

\subsubsection{Conclusions regarding the relative performance of the ETD}

The performance of the ETD algorithm depends on the SNR value of pulses. When the detection threshold is set to a large value such as $8 \sigma$, the ETD algorithm is capable of detecting $80.5 \%$ of all pulses detected by the conventional algorithm. When the detection threshold is set to $5 \sigma$, the 
ETD algorithm is able to detect only $40.4 \%$ detectable by the conventional algorithm.

As it is difficult to evaluate the exact value of $[\mathrm{SNR}$ of pulses parameterized by a low $[\mathrm{SNR}]$ value, we set the lower threshold to $S \mathrm{SNR}=5$. Note that the ETD algorithm is able to detect many pulses with the conventional $\mathrm{SNR}$ below 5 due to its ability to detect pulses with broad pulse profiles. For the conventional algorithm, however, setting the SNR threshold to 4 or smaller leads to detecting hundreds or even thousands of candidate pulses, which will further require manual inspection to confirm or reject a pulse. Also, the pulses with $\mathrm{SNR}$ lower than 4 are not significant in time series and hard to confirm by eye. This is why the conventional algorithm can not be used to confirm single candidate pulses detected by the ETD algorithm, when $\mathrm{SNR}$ is set to a value below 5 . If we assume that all single candidate pulses detected by the ETD algorithm are due to noise, in this case the average false alarm rate of the ETD algorithm is equal to $72.5 \%$. The average FAR of the conventional algorithm is $68.8 \%$.

\subsubsection{Using the MF-SFT Algorithm to Confirm Candidates}

Since the conventional algorithm can not confirm some single candidate pulses detected by the ETD algorithm, we used a third method called Matched Filtering in Spatial Fourier Transform domain (MF-SFT), to confirm those candidate pulses.

The MF-SFT algorithm detects all pulses with the $\mathrm{SNR}$ values greater 7 . When the $\mathrm{SNR}$ is set to 5 and above, the MF-SFT algorithm detects $89.4 \%$ previously detected by the conventional algorithm. This indicates that the detection ability of the algorithm is relatively accurate.

The percentage of single candidate pulses detected by the ETD algorithm confirmed by the MF-SFT algorithm is $79.1 \%$. If we assume that the MF-SFT is a baseline, then the ETD algorithm demonstrates a relatively good performance.

\subsection{Limitations of the ETD algorithm}

The main drawback of the ETD algorithm is its decision rule (step 3 of the algorithm). If the candidate's ETD value is greater than maximum ETD value of noise in both the $16 \times 16$ and $32 \times 32$ matrix, we decide the candidate is a pulse. Otherwise, we decide the candidate is noise. Since different RRATs have different DM values and other parameters, testing both windows is 
not appropriate, only one optimal window size should be used for a specific RRAT. As of now, the decision rule is too stringent and it often leads to missed pulses.

\subsection{Future Work}

In the future we would like to continue testing performance of the ETD algorithm. The algorithm can be applied to other data containing isolated astrophysical pulses. For example, the ETD algorithm can be applied to search for Fast Radio Bursts or to search for perytons. We would also like to vary a number of parameters of the ETD algorithm. For example, we can choose different sizes of windows in the SFT space and find the maximum value of the ETD metric over all selected window size.

Also, it would be interesting to see how the ETD algorithm would perform when applied to beamformed array data (such as obtained by the Focal L-band Array instrument recently installed at the Green Bank Telescope) and how it compares to the conventional algorithm in this case. 


\section{Bibliography}

[1] "frbsearch directory." http://astro.phys.wvu.edu/frbsearch/.

[2] "The rratalog." http://astro.phys.wvu.edu/rratalog/.

[3] D. R. Lorimer and M. Kramer, Handbook of pulsar astronomy, vol. 4. Cambridge University Press, 2005.

[4] M. A. McLaughlin, A. Lyne, D. Lorimer, M. Kramer, A. Faulkner, R. Manchester, J. Cordes, F. Camilo, A. Possenti, I. Stairs, et al., "Transient radio bursts from rotating neutron stars," Nature, vol. 439, no. 7078, pp. 817-820, 2006.

[5] M. Keith, R. Eatough, A. Lyne, M. Kramer, A. Possenti, F. Camilo, and R. Manchester, "Discovery of 28 pulsars using new techniques for sorting pulsar candidates," Monthly Notices of the Royal Astronomical Society, vol. 395, no. 2, pp. 837-846, 2009.

[6] E. Keane, M. Kramer, A. Lyne, B. Stappers, and M. McLaughlin, "Rotating radio transients: new discoveries, timing solutions and musings," Monthly Notices of the Royal Astronomical Society, vol. 415, no. 4, pp. 3065-3080, 2011.

[7] S. Larsson, "Parameter estimation in epoch folding analysis," Astronomy and Astrophysics Supplement Series, vol. 117, no. 1, pp. 197-201, 1996.

[8] S. P. Reynolds, K. J. Borkowski, B. M. Gaensler, N. Rea, M. McLaughlin, A. Possenti, G. Israel, M. Burgay, F. Camilo, S. Chatterjee, et al., "Discovery of the X-ray counterpart to the rotating radio transient j1819-1458," The Astrophysical Journal Letters, vol. 639, no. 2, p. L71, 2006.

[9] M. Burgay, M. McLaughlin, and S. Reynolds, "On the debated nature of rotating radio transients," in AIP Conference Proceedings, vol. 924, pp. 607-612, AIP, 2007.

[10] X.-D. Li, "On the nature of part-time radio pulsars," The Astrophysical Journal Letters, vol. 646, no. 2, p. L139, 2006.

[11] Q. Luo and D. Melrose, "Pulsar radiation belts and transient radio emission," Monthly Notices of the Royal Astronomical Society, vol. 378, no. 4, pp. 1481-1490, 2007.

[12] J. M. Cordes and R. Shannon, "Rocking the lighthouse: circumpulsar asteroids and radio intermittency," The Astrophysical Journal, vol. 682, no. 2, p. 1152, 2008. 
[13] P. Weltevrede, B. Stappers, J. Rankin, and G. Wright, "Is pulsar b0656+ 14 a very nearby rotating radio transient?," The Astrophysical Journal Letters, vol. 645, no. 2, p. L149, 2006.

[14] R. N. Manchester, A. G. Lyne, F. Camilo, J. Bell, V. Kaspi, N. D’Amico, N. McKay, F. Crawford, I. Stairs, A. Possenti, et al., "The parkes multi-beam pulsar survey-i. observing and data analysis systems, discovery and timing of 100 pulsars," Monthly Notices of the Royal Astronomical Society, vol. 328, no. 1, pp. 17-35, 2001.

[15] “40 years of dish.” http:wWw.abc.net.au/science/articles/2010/02/09/2814525. htm.

[16] R. C. Gonzalez and R. E. Woods, "Digital image processing prentice hall," Upper Saddle River, NJ, 2002.

[17] C. M. Stein, "Estimation of the mean of a multivariate normal distribution," The annals of Statistics, pp. 1135-1151, 1981.

[18] D. L. Donoho and J. M. Johnstone, "Ideal spatial adaptation by wavelet shrinkage," biometrika, vol. 81, no. 3, pp. 425-455, 1994.

[19] B. Zackay and E. O. Ofek, "An accurate and efficient algorithm for detection of radio bursts with an unknown dispersion measure, for single dish telescopes and interferometers," The Astrophysical Journal, vol. 835, no. 11, p. 13, 2017.

[20] N. A. Schmid and R. M. Prestage, "Blind detection of isolated astrophysical pulses in the spatial fourier transform domain," Monthly Notices of the Royal Astronomical Society, 2018.

[21] "Sigproc." http://sigproc.sourceforge.net/

[22] M. McLaughlin, A. Lyne, E. Keane, M. Kramer, J. Miller, D. Lorimer, R. Manchester, F. Camilo, and I. Stairs, "Timing observations of rotating radio transients," Monthly Notices of the Royal Astronomical Society, vol. 400, no. 3, pp. 1431-1438, 2009. 


\section{Appendix A}

\section{Appendix}

To better explain the choice of maxval, we will use a rectangular function as a profile of a pulse. Let $f(x)$ be a rectangle function:

$$
f(n)= \begin{cases}1, & |n|<\frac{W}{2} \\ 0, & |n| \geq \frac{W}{2}\end{cases}
$$

the discrete Fourier Transform of A.1, denote it as $F(u)$, is given as

$$
F(u)=\sum_{n=-W / 2}^{W / 2} \exp \left(-j \frac{2 \pi u}{N} n\right)=\frac{\sin (\pi u W / N)}{\sin (\pi u / N)}=W \frac{(u W / N)}{(u / N)}
$$

for $u=0, \ldots, N-1$.

Thus the discrete Fourier Transform of a rectangular function is a ratio of two functions. The first zero of the in the numerator occurs at $u=N / W$. The first zeros of the denominator is at $N$. Note that the main lobe of the in the numerator contains most of the energy of the signal. Thus maxval in our computations has to be approximately set to $N / W$. For example, if $N=1024$ and $W=32$, then $N / W=32$. Using the typical sampling interval of length $10^{-4}$ seconds of the Parkes telescope, the width of the pulse detectable using maxval of 32 is $3.2 \mathrm{~ms}$. 